\title{
Checklist of the Amphipoda (Crustacea) from continental waters of Russia, with data on alien species
}

\author{
Контрольный список Amphipoda (Crustacea) континентальных \\ вод России, со сведениями о чужкеродных видах
}

\author{
V.V. Takhteev ${ }^{1}$, N.A. Berezina ${ }^{2}$, D.A. Sidorov ${ }^{3}$ \\ B.B. Тахтеев ${ }^{1}$, Н.А. Березина ${ }^{2}$, А.А. Сидоров ${ }^{3}$
}

${ }^{1}$ Scientific Research Institute of Biology and Department of Biological and Soil Sciences, Irkutsk State University, Karl Marx St. 1,
Irkutsk 664003, Russia. Е-mail: amphipoda@yandex.ru
${ }^{1}$ Научно-исследовательский институт биологии и кафедра зоологии беспозвоночных и гидробиологии Иркутского государ-
ственного университета, ул. К. Маркса, 1, Иркутск 664003, Россия. E-mail: amphipoda@yandex.ru
${ }^{2}$ Zoological Institute of the Russian Academy of Sciences, Universitetskaya Nab. 1, St. Petersburg 199034, Russia. E-mail:
nadezhda.berezina@zin.ru
${ }^{2}$ Зоологический институт Российской академии наук, Университетская наб., 1, Санкт-Петербург 199034, Poccия. E-mail:
nadezhda.berezina@zin.ru
${ }^{3}$ Institute of Biology and Soil Science of the Russian Academy of Sciences, Far Eastern Branch, Prospekt 100-Let Vladivostoku 159,
Vladivostok690022, Russia. Е-mail: biospeorossica@gmail.com
${ }^{3}$ Биолого-почвенный институт Дальневосточного отделения РАН, проспект 100 лет Владивостоку, 159, Владивосток 690022,
Россия. Е-таil: biospeorossica@gтаil.сот

KEY WORDS: amphipods, taxonomy, nomenclature, biodiversity, distribution, biogeography, alien species.

КЛЮЧЕВЫЕ СЛОВА: амфиподы, таксономия, номенклатура, биоразнообразие, распространение, биогеография, чужеродные виды.

ABSTRACT. A checklist of the amphipod fauna from continental water bodies, streams and subterranean waters of the Russian Federation is provided (based on data for the end of 2013). Species are divided into 11 ecological and biogeographic groups: Holarctic, West Palearctic, Baltic Sea Estuarine, Siberia-Pacific Coast (East Palearctic), Amphi-Pacific, Caucasus, Central Asiatic and Baikalian, and three groups of escapees (emigrants) from the Baikalian, Ponto-Caspian and Arctic Oceans. Twenty-six families, 110 genera and 581 species and subspecies are reported. Species that constitute the autochthonous complex of Lake Baikal comprise $61 \%$ of the fauna (276 species, and 78 subspecies). The current taxonomic and the nomenclatural problems of amphipods from the Baikalian and Caspian groups are discussed. A separate list of alien species (or invaders) for continental waters of Russia is provided.

РЕЗЮМЕ. Приведен таксономический контрольный список видов амфипод, зарегистрированных в континентальных водоемах, водотоках и подземных водах Российской Федерации (по данным на конец 2013 г.). Виды подразделены на 11 эколого-биогеографических групп: голарктические, западно-палеарктические, балтийские эстуарные, сибирско-тихоокеанского региона (восточно-палеарктические), амфипацифические, кавказские, центрально-азиатские, байкальские, эмигранты из Байкала, виды понто-каспийского происхождения, эмигранты из Северного Ледовитого океана. На дан- ный момент отмечено 26 семейств, 110 родов и 581 вид и подвид амфипод. На виды, составляющие автохтонный комплекс озера Байкал, приходится $61 \%$ состава фауны (276 видов и 78 подвидов). Даны пояснения, касающиеся современных проблем таксономии и номенклатуры байкальских и каспийских амфипод. Отдельно приведен список чужеродных видов, или видов-вселенцев в континентальные водоемы регионов России вне их первоначального ареала.

\section{Introduction}

A faunal inventory of our planet remains an urgent task. A basic taxonomic knowledge is necessary for bio-evolutionary studies as well as for understanding biogeography. The Amphipoda are one of the most successful and rapidly evolving malacostracan orders, displaying a tremendous diversity in marine, terrestrial and continental waters. We attempt to list the amphipods inhabiting the continental waters of the Russian Federation.

Our biogeographic analysis was limited to the borders of the Russian Federation (as of 2013). There are several reasons why we did not distinguish natural geographic areas. First, there is no generally accepted biogeographic regionalization scheme for continental waters. For example, Northern Eurasia can belong either to the Palearctic region or to the Holarctic region. Furthermore, the Palearctic southern boundary is de- 
termined in several different ways. Second, historically, biogeographic analysis of the amphipod fauna was confined to separate regions. For example, the amphipod fauna of the Russian Altai Mountains is considered relatively well studied [Martynov, 1930] while the Mongolian Altai still remains a "blind spot" and we can only presume that the Altai Mountain fauna has a certain historical unity.

We summarize the fragmentary biogeographical data on the amphipod fauna in different regions and discuss the current taxonomy, taking into account different points of view. We refer to a number of relevant taxonomic studies on Russian amphipods in order to increase international readers' awareness of the little known "Russian literature".

In addition, we analyse the range extension and the current biogeographic state of alien species (invaders) among amphipods in continental waters of Russia. In the frame of this paper we use the term "alien species" to specify the taxa that spread beyond their historically native range over the last 100 years, or species introduced to a new range where they established themselves and spread over the region. The introduced species are a special case of alien species, namely the species that have been transported due to human activities, either intentionally or accidentally, to a region in which they did not occur in historical times and are now reproducing in the wild [Jeschke, Strayer, 2005].

In Russia with its extensive territory (the total area measuing 17075 thou $\mathrm{km}^{2}$ ), the donor regions for alien species are other regions of the same country or other countries and continents. For separate eurybiotic species of amphipods that have capacity for active migrations in rivers to considerable distances, and for rapid reproduction, the distribution rate is high. The majority of alien amphipods penetrated the Baltic Sea basin from the basins of the Volga River, Caspian Sea, Black Sea and the Sea of Azov after the construction of artificial canals, reservoirs and drainage systems and the formation of waterways (canal-river network). The Volga-Don, Volga-Baltic, Dnieper-Vistula and DanubeRhine systems are the most important waterways for the dispersal of amphipods over the European continent from the Ponto-Caspian basin to the Baltic Sea (for details, see: [Berezina, 2007a]).

\section{Material and methods}

All species from the continental waters of Russia, with the exception of the Caspian and Aral Lakes (derivatives of the ancient Tethys Ocean), were included. However, alien species that originated from the Caspian Sea and naturalized in other regions of Russia were included. It should be noted that we avoid the word "freshwater amphipods", and use "amphipods of continental waters" because some amphipod species occur in brackish-water estuaries, salt lakes and mineral springs. Also, some species are able to be tolerant and reproduce successfully over a wide range of salinity. For example, Gammarus lacustris Sars, 1863 is report- ed for salinities ranging from 0.3 to $25 \mathrm{~g} / 1$ [Takhteev, 2009]. On the other hand, we have not included on the list stenohaline marine species, such as Marinogammarus finmarchicus (Dahl, 1938) or Spasskogammarus spasski Bulycheva, 1952, although they were found in the mouths of creeks, rivers, and in the upper horizons of intertidal and subtidal zones, at salinities above 5\% $(5 \mathrm{~g} / \mathrm{L})$. This boundary was accepted by the Venice System for the Classification of Marine Waters (1958) as the upper border of freshwater origin fauna distribution [Khlebovich, 1974].

The genera and species lists are based on valid families; all controversial cases will be mentioned in the text where appropriate. In the generic list we affiliate each specific genus to one of the three ecological complexes (Paleolimnic, Mesolimnic and Neolimnic) which was originally proposed for Lake Baikal fauna [Martinson, 1967] and later extended to all inhabitants of continental waters [Starobogatov, 1970; Baikalogy, 2012]. Each complex was referred to the time of isolation from marine ancestors, the duration of which conditionally determines its ecological features. It should be noted that we highlight here the age of all three complexes, although our views disagree with the view maintained by Martinson [1967] and Starobogatov [1970] (see below).

The "Paleolimnic complex" includes the ancient freshwater inhabitants, separated from the marine relatives in the Mesozoic era (Jurassic, Cretaceous). For example, the underground amphipod family Crangonyctidae is attributed to the Paleolimnic complex, taking into account their amphiboreal distribution in Eurasia and North America.

The "Mesolimnic complex" derives from the marine ancestors in the early Cenozoic era, from the Paleocene to the Oligocene. In particular, freshwater species of the genus Gammarus belong to this complex as derivatives of the Tethys Ocean period [Hou et al., 2011] as well as all endemic amphipods of Lake Baikal [Starobogatov, 1970; Takhteev, 2000b; Baikalogy, 2012].

The "Neolimnic complex" is the youngest, from the late Cenozoic era, and relates to Miocene, Pliocene, Pleistocene and, probably in some cases, to the Holocene. The distribution of the Neolimnic complex species is restricted to areas of marine transgression during these periods, such as the lakes and river estuaries of the Arctic Ocean basin, and coasts and islands in the Asian-Pacific part of Russia. For example, this complex includes representatives of the families Gammaracanthidae, Pontoporeiidae, Oedicerotidae, Uristidae, Anisogammaridae and Hyalidae. Also, species of Ponto-Caspian origin, inhabiting the continental waters within the specified period, were attributed to the Neolimnic complex.

The zoogeographical division of Russian continental water bodies based solely on the Amphipoda creates difficulties in choosing the guiding principle. Initially, we relied on the geography and origin of the taxa. In this case, the construction of a single zoogeog- 


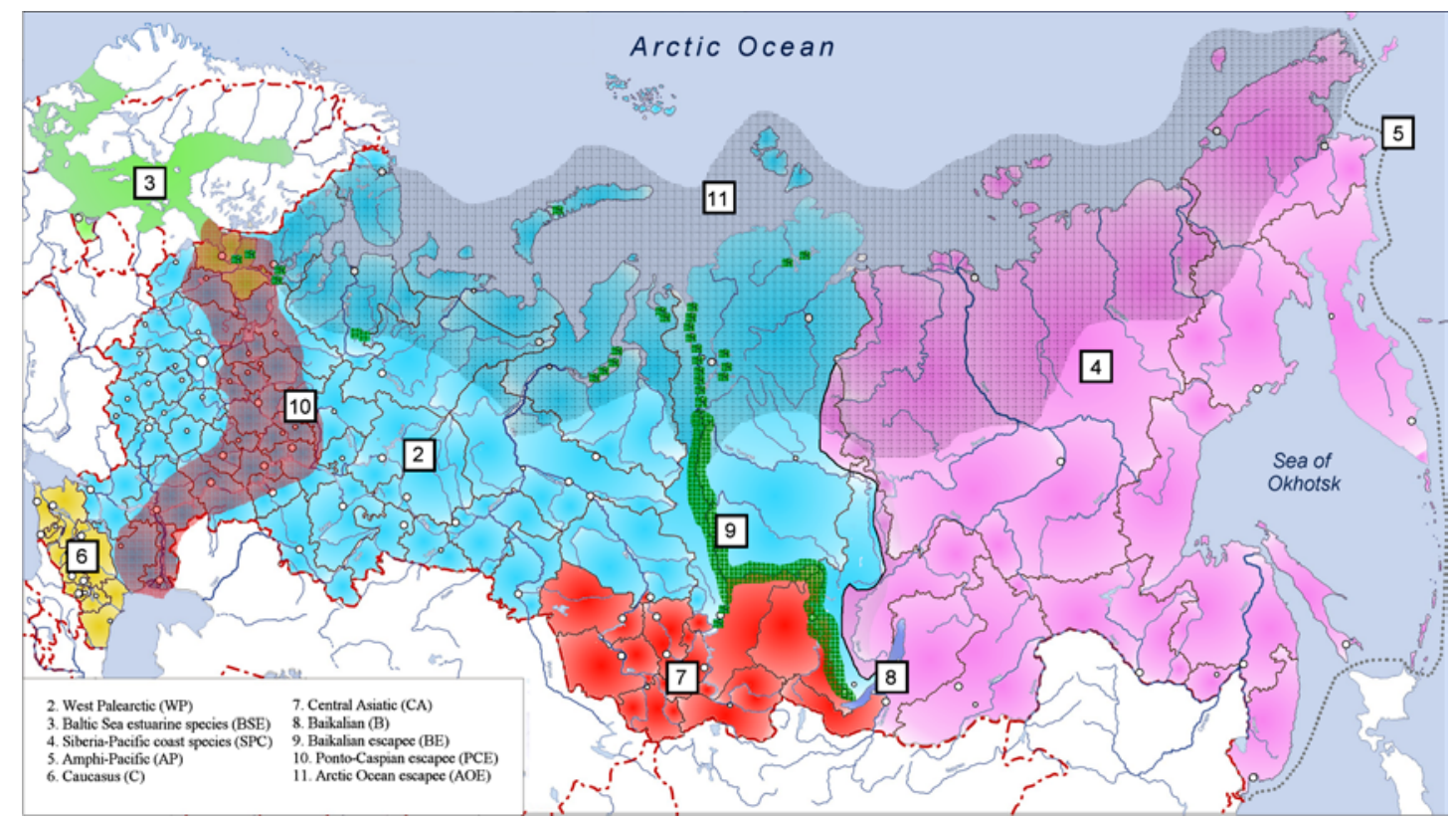

Fig. 1. Map displaying the distribution of the biogeographic groups of amphipod species in Russia. Group 1 "Holarctic" $(\mathrm{H})$ is not shown; it occurs throughout the territory.

Рис. 1. Карта, отображающая распространение биогеографических групп видов амфипод в России. Замечание: группа № 1 “Голаркты” (Holarctic, H) не показана, распространена по всей территории.

raphy for subterranean and epigean species does not make sense, because it will be artificial and can be realized only on the supra-genus levels. In general, we are committed to the view of Darlington, who defined three zoogeographical classes [Chernov, 1975]. Therefore, to obtain a qualitative picture of the fauna, a number of species groups have been allocated based on their present geographical distribution and partly on centers of origin (Fig. 1). Eleven amphipod species groups were distinguished for the biogeographic analyses within this paper.

1. Holarctic $(\mathbf{H})$. Species distributed in the Northern Hemisphere on both continents, such as the limnophilic Gammarus lacustris and the alien Gammarus tigrinus Sexton, 1939, are referred to this group.

2. West Palearctic (WP). The group includes species which are wide-spread in Europe and/or Asia but not farther east than the west coast of Lake Baikal in the south and the Taimyr Peninsula in the north. The Baikal mountain system and Lake Baikal is an important zoogeographic barrier for many aquatic and terrestrial faunal groups. Taimyr is the watershed between the basins of the majority of Siberian rivers including the $\mathrm{Ob}$ ' and Yenisei rivers on the west and the Lena river on the east of Russia.

3. Baltic Sea estuarine species (BSE). The species from the Baltic Sea region were distinguished as a separate group because the Baltic Sea is the unique ecosystem, being only slightly saline (brackish). The Mediterranean and the Ponto-Caspian origin species including Corophium volutator (Pallas, 1766), characterized by an Amphi-Atlantic distribution, were placed in this group.
4. Siberia-Pacific coast species (SPC). This complex includes species from the Russian Far East and Eastern Siberia, which extend west not further than eastern coast of Lake Baikal and the Lena river basin.

5. Amphi-Pacific (AP). This group includes species from both sides of the Pacific Ocean and includes only one terrestrial amphipod Traskorchestia ochotensis (Brandt, 1851).

6. Caucasus (C). Only endemic species from the genera Gammarus, Niphargus, Lyurella and Synurella were found in freshwater ecosystems of the Caucasus.

7. Central Asiatic (CA). Species inhabiting the mountain zones of Central Asia. Several species of Gammarus are endemic to specific mountain systems. Asian species of the genus Stygobromus (mainly North American), tending to the mountain zones of the Altai and Baikalian Siberia, are also referred to this group.

8. Baikalian (B). All species endemic and subendemic to Lake Baikal are included.

9. Baikalian escapee (BE). This group consists of species of Baikalian origin that were found in the Angara and Yenisei rivers, and rarely in northern latitudes including the $\mathrm{Ob}$ ' river estuary and northwestern Russian lakes but not in Lake Baikal. In particular, the "glacial relicts" Pallasea quadrispinosa Sars, 1867 and Pallasea laevis Ekman, 1923 belong to this group. The former species inhabits the Northern regions of both the European and Asian parts of the country (see Fig. 1, group 9), and the status of the latter species needs to be tested; it was found on the Novaya Zemlya archipelago and recently (presumably) in the cave waters in the North of European Russia [Sidorov et al., 2011]. 
10. Ponto-Caspian escapee (PCE). The species within this group originate from the basins of the Caspian and Black Seas and the Sea of Azov including the lower courses of large rivers emptying into the seas. This group includes species from the upstream migratory complex in the rivers of the Sea of Azov and the Caspian and Black Seas (Volga, Kama, and Don), and all Ponto-Azov-Caspian origin species that have penetrated the Baltic Sea basin in connection with the construction of artificial canals, and with other humanmediated vectors. Species endemic to the Sea of Azov and the Black and Caspian Seas are not included in this group and on the checklist.

11. Arctic Ocean escapee (AOE). This group includes relict species of the late Cenozoic marine transgressions, such as Gammaracanthus spp., and Monoporeia affinis (Lindström, 1855). In the literature, they are often named the "glacial relicts", but this is not quite correct. The transgressions of the ocean to land did not occur during periods of glaciations; they tok place at interglacial periods due to an increase in water mass runoff to the ocean, and the thermal expansion of the water. Some views of the existence of periglacial dammed lakes that could serve as the natural "gateways" for marine fauna elements [Grosswald, 1998, etc.] are still a disputed topic.

\section{Taxonomic notes}

The amphipod genus Gammarus is characterized by a variety of species amongst the Palearctic groups. The number of new species continues to grow. In the summary of Barnard \& Barnard [1983] there were 117 species, mostly freshwater. According to Hou et al. [2013], the total number of species is more than 200. The genus needs further revision; given the fact that parallel speciation in different mountain ranges of Asia from the same ancestral forms may be assumed. On the other hand, we have attempted to unite all species recorded in Russia within three groups according to Stock [1967] and Karaman \& Pinkster [1977].

A difficult situation has arisen with the taxonomy of Lake Baikal amphipods. A partial revision was presented by Bousfield [1977], Barnard \& Barnard [1983] and Takhteev [2000b, 2012]. One of us (Takhteev, V.V.) described 32 new species and subspecies and made redescriptions for more than 40 taxa based primarily on the type material (see Takhteev, 1992a, b, 1993, 1995, 1997, 1999a, 2000a, b; Takhteev, Levashkevich, 2006). However, a series of publications by Kamaltynov [1999, 2001, 2009] have consistently introduced new families and subfamilies, the "old" genera were fragmented into a number of a new genera, and numerous subspecies of amphipods (totaling 65) were elevated to distinct species. As a result, the system proposed by R.M. Kamaltynov contains 10 families and 13 subfamilies of amphipods (excluding nominative). In addition, 19 new genera (excluding replacement names) were established, and 12 subgenera were assigned to generic rank by Kamaltynov, almost without any taxonomic evidence or analyses for the proposed changes [Kamaltynov, 2001, 2009]. He has not provided keys to the genera and species; as a result, the new taxa are difficult to determine.

Kamaltynov [2001] proposed four new names, established from the undefended elevation to species level of the previously known intraspecific variations that were never described properly. For two of these four species (Micruropus stelleri Kamaltynov, 2001 and $M$. tomilovi Kamaltynov, 2001) he referred to the drawings of the separate body appendages in a paper of Bazikalova [1962] and gave to these figures the status of holotype [sic!]. And it is even not known these figures refer to the same specimen. The third species, Hyalellopsis linevichae Kamaltynov, 2001, was established by reference to the description of Hyalellopsis tixtonae Sowinsky, 1915 in a monograph by Bazikalova [1945], in the belief that she, under this name, incorrectly described the new taxon. A review of the type material $H$. tixtonae indicates that Sowinsky [1915] and Bazikalova [1945] described the same form, and the name $H$. linevichae is a nomen nudum. For the fourth species, Carinurus karamani Kamaltynov, 2001, the author mentions a single specimen (syntype), but its description is not provided. In this case, Kamaltynov referred briefly to the differences of "Carinurus belkini forma A" in Bazikalova [1935, 1945]. As no type material was provided, and no proper descriptions or diagnoses prepared, we consider none of these names to be valid. Kamaltynov also revised the Caspian Sea fauna and created two new families: Behningiellidae and Iphigenellidae. The justification for the new families is brief [Kamaltynov, 2001], and his definitions imply that the revision by the previous authors was incomplete.

Similarly, we also cannot agree with the proposal of Kamaltynov [2001, 2009], considering 65 subspecies of Lake Baikal amphipods to be an independent species. These nomenclatural changes have been made by "a package or a horde", based on the facts (sometimes single) of co-occurrence of a different subspecies of a single species in the same samples. Unfortunately, this is already reflected in the WoRMS database (http:/ /www.marinespecies.org/aphia.php? $\mathrm{p}=$ taxdetails\&id =720708). We are firmly confident that every single case of a change of rank of the taxon requires a special justification. Suffice it to say that Dybowski [1874] described many subspecies in general as varieties (var.). In the subspecies, they were included for formal reasons, following the first edition of the International Code of Zoological Nomenclature [1961], where all pre-existing variations were given the subspecies status. Bazikalova [1945] described taxa as subspecies in the presence of a series of transitional forms to the nominative subspecies. Hence, without a specific justification for the change, we continue to consider the subspecies from the Lake Baikal in the same rank to be valid. 
It is necessary to adhere to the principle of conservation and promote stability of the existing system, as long as there are no compelling reasons to change it. There is also a clear need to assign criteria for systematization of families and genera. One of the authors (Takhteev, V.V.) believes that a taxonomic revision of a polymorphic group, such as amphipods of Lake Baikal, must use the archaetypical method [Takhteev, 2000a, 2010, 2012] (see also Lyubarsky, 1996, pp. 12, 69-72, 102-107). Since the morphological boundaries of many taxa (families and genera) intergrade, they can only be distinguished from one another with difficulty, whereas some species and genera possessing a sharply deviating character must be allocated as the archetype ("core" of the taxon). A taxonomic diagnosis must be based on the main plan of morphology of the archetype form. A taxonomic rank identical with the "core" should not be assigned for peripheral and aberrant forms. Otherwise there arises an artificial inflation of taxa where many taxa are fragmented, creating an abundance of "dwarf" taxonomic groups of the highest rank, and the ranks themselves become worthless. All taxonomic ranks above the species level are inclusive groups, whereas only the species rank is exclusive. For example, there is no need to placethe the species Baikalogammarus pullus (Dybowsky, 1874) in the monotypic family Baikalogammaridae Kamaltynov, 2001. This is a peripheral group in the family Micruropodidae, and its only sole representative is characterized by archetypical features of this family, such as the small size, lack of cuticular armature and spines on urosome, one article in accessory flagellum of antenna 1 as well as other shared features [Takhteev, 2012].

In this publication, we do not discuss the macrosystematics of the Baikalian amphipods in detail but emphasize highlight that Takhteev's [2000b] system was adopted. On the other hand, from the perspective of the archaetypical approach we agree with some proposals made by Kamaltynov [2001], such as the establishment of the family Micruropodidae (but its composition was revised, and some of the taxa were assigned to the family Carinogammaridae Tachteew, 2000), and the subfamilies Hyalellopsinae and Parapallaseinae [Takhteev, 2010, 2012]. We also recognize some new genera proposed by Kamaltynov, such as genus Profundalia, and the two subgenera Babr and Dorogostajskia.

The genus Profundalia Kamaltynov, 2001 includes only one species P. tenuis, which Bazikalova [1971] decided to exclude from the genus Eulimnogammarus, but did not specify to which genus it is belong. This species has a small size (less than $5 \mathrm{~mm}$ ), one-articulate outer ramus on uropod 3, and lacks cuticular armature on the body segments and spines on urosome, which is typical for the family Micruropodidae. On the other hand, in the accessory flagellum of antenna 1 there are up to four articles, and antenna 1 is not smaller than the body length. We believe that $P$. tenuis is among the species with an unclear taxonomic status.

The genus Babr Kamaltynov et Väinölä was reported in Kamaltynov [2001]; later, it was characterized in detail by Daneliya et al. [2009]. They transferred Pallasea (Pallasea) baikali baikali and Pallasea (Pallasea) baikali nigromaculata to the aforementioned genus, and the subspecies Pallasea (Pallasea) baikali inermis was synonymized with Babr baikali. In our opinion, the taxonomic problem of Pallasea needs additional studies involving both morphological and molecular methods. In particular, Pallasea baikali inermis is a local endemic subspecies which inhabits shallow waters of the Selenga river delta. The morphological differences of this endemic subspecies are, probably, the result of specific environmental conditions in the delta area and it may be genetically identical with the nominative subspecies. In this paper, we accept Pallasea as a single genus but with four well-defined subgenera, namely Pallasea, Babr, Homalogammarus and Pentagonurus.

Dorogostajskia Kamaltynov, 2001 was introduced as a substitute name for Spinacanthus Dorogostajsky, 1930 nomen praeoccupatum [Kamaltynov, 2001]. We regard Dorogostajskia as a subgenus of Brandtia due to the presence of transitional forms between these taxa (Brandtia (Dorogostajskia) insularis). Bazikalova [1948] assigned Spinacanthus (currently Dorogostajskia) to the subgenus rank because of this. However, Kamaltynov [1992, 1999] returned this taxon to the genus rank again, and then identified some of the species as a discrete genus Dedyuola [Kamaltynov, 2001]. Clearly, the status and composition of this genus needs further attention. We support the assumption that Dorogostajskia is a subgenus, and a recent revision of Brandtia (Dorogostaiskia) parasitica (Dybowsky, 1874) as consisting of five subspecies [Daneliya, Väinölä, 2014] is reflected in the checklist.

The remaining taxa proposed by Kamaltynov are poorly substantiated and not included on our list as well as some Baikalian species designated in publications as the new species but have not been formally described. Two families established by Kamaltynov for the Caspian amphipods (Behningiellidae and Iphigenellidae) based on published data are included on the list but their status needs verification on the original materials.

The status of the Baikalian endemic genus Hyalellopsis Stebbing, 1899 with four subgenera (Hyalellopsis s.str., Boeckaxelia Bazikalova, 1948, Dorogammarus Bazikalova, 1945 and Gammarosphaera Bazikalova, 1936) requires further clarification. We support such a subdivision according to Bazikalova [1948] but with the only difference that it remains independent of Gammarosphaera (Bazikalova synonymized Gammarosphaera with Hyalellopsis). Perhaps, the subgenus Boeckaxelia should include Cheirogammarus inflatus [Sowinsky, 1915]. However, the only available specimen of $C$. inflatus (holotype) is dissected, and the status of this species remains unclear.

To specify the type species of some Baikalian genera, the following fact must be taken into account. Opinion 105 of the International Commission of Zoological Nomenclature withdrew the work of Dybowski 
[1926/1927], but about the publication [Dybowski, 1924] nothing is said, i.e. it should be considered valid. Also, Opinion 105 proclaims only the new generic names of Dybowski invalidated: "That all of the new names published in Dybowski's paper, "Synoptisches Verzeichnis mit kurzer Besprechung der Gattungen und Arten dieser Abteilung der Baikalflohkrebse" (Bul. internat. Acad. polonaise d. Sci. et d. Lettres, 1926, No. 1-2b, Jan.-Feb., pp. 1-77), are hereby suppressed under Suspension of the Rules on the ground that the application of the Rules in accepting them "will clearly result in greater confusion than uniformity".

At the same time, Dybowski [1924, 1926] designated the type species for a number of the genera proposed by other authors. Taking into account that paper published in 1924, it has not been formally rejected and these designations should be recognized. This explains the difference in indication of the type species on our list and other authors [Bazikalova, 1945; Kamaltynov, 1992, 1999, 2001]. According to Dybowski [1924], all subsequent designations should be considered invalidated, except where Dybowski [1924] unnecessarily renamed the genera.

It should also be recognized that the taxonomy of the genera Chaetogammarus and Echinogammarus is also confused and is still far from being clearly determined. Karaman [1977] synonymized some formerly used generic names (Marinogammarus Schellenberg, 1937, Chaetogammarus Martynov, 1924) with the name Echinogammarus Stebbing, 1899 uniting all gammarid species with a scale-like endopodite of uropod 3. This view was supported later by Karaman \& Barnard [1979] and Barnard \& Barnard [1983] and the name Echinogammarus was used for E. ischnus until 1995. Stock [1995] discussed generic diagnoses of the two genera and suggested that those species almost completely lacking setation on the pereiopods, urosome, coxal plates and on the ventral margins of the epimeral plates belong to Chaetogammarus and those with the presence of longer setae on the same parts belong to Echinogammarus. Stock [1995] returned to the name Chaetogammarus for a group of taxa of Echinogammarus sensu Karaman, restricting this last name only to the Echinogammarus berilloni group [Pinkster, 1973].

Finally, we realize that the checklist and the system of supraspecific taxa presented is, to some extent, these author's viewpoint; it may contain some shortcomings and should be discussed and developed further.

\section{Data composition and designations}

Throughout the paper information is provided and structured in the following manner:

Table 1: The hierarchical taxonomic position of the genera (taxa of the subfamily rank are mentioned), indicating the following data:

- number of valid species and subspecies in each genus (in parentheses)

- eco-zoogeographical characteristic of the genera: p - paleolimnic (taxa have no presumptive marine ancestors; synonym - Old Limnetic), $\mathbf{m}$ - mesolimnic (taxa with distant marine ancestors), and $\mathbf{n}-$ neolimnic (taxa with recent marine ancestors).

Table 2: Checklist in alphabetic order (taxa of the subgenus rank indicated, consisting of the species names). Within the checklist we provide:

- valid species and genus names with author and year;

- list of principal species synonyms;

- (original combination) of binomial species name if different from the present one;

- type species of genera shown by ' $\bullet$ ', if the type species is absent in Russia, it is indicated separately;

- fixation of type species indicated in [brackets];

- taxon described erroneously or needing re-examination (doubt about validity) shown by the asterisk '*';

- taxon probably extinct - ' $\dagger$ '.

\section{List of alien amphipod species of Russia}

The dispersal rate of alien amphipods, and also of many other groups of organisms is often associated with human activities. In different cases, the factors can act additionally or antagonistically with one another or with natural processes. Destruction of natural barriers between different basins of Europe in the $19^{\text {th }}$ and $20^{\text {th }}$ centuries is considered to be one of the most important factors, which has resulted in range expansion of many species in different directions from southern basins of the Volga River, the Caspian Sea, the Black Sea and the Sea of Azov to European Russian continental waters situated in more northern regions. The case study area in the European Russia encompasses large lakes, namely Ladoga, Onega, Peipsi and Il'men', reservoirs and small water bodies of the Volga, Kama and Don river basins as well as the rivers flowing into the Baltic Sea (Neva and Narva), the Curonian and Vistula lagoons and the associated reservoirs of Kaliningrad oblast.

During the 1950s-1980s, large-scale intentional introductions of crustaceans were a major vector of amphipod invasions in inland waters of European Russia and Siberia. Enrichment of fish production was the principal motivation for the introductions. The largescale mass transportations of amphipods (even unknown species), conducted in the former U.S.S.R. during the second part of the last century, were often not documented, which made it difficult to determine the invasion routes. It is known that at least 30 amphipod species of Ponto-Caspian origin, three species of Baikalian origin (Micruropus possolskii, M. wohlii, and Gmelinoides fasciatus), and formely so-called "glacial relicts" (Arctic Ocean escapee in the present study), such as Monoporeia affinis and Pallasea quadrispinosa, were used for intentional introductions through the former U.S.S.R. area [Gordeev, 1954; Greze, 1958; Ioffe, 1968; Zadoenko et al. 1985; Berezina, 2007a]. 
Order Amphipoda Latreille, 1816

Suborder Gammaridea Latreille, 1802

Superfamily Crangonyctoidea Bousfield, 1973

Family CRANGONYCTIDAE Bousfield, 1973

Amurocrangonyx Sidorov et Holsinger, 2007

Crangonyx Bate, 1859

(1)

Lyurella Derzhavin, 1939

Stygobromus Cope, 1872

Synurella Wrzesniowski, 1877

Family NIPHARGIDAE Bousfield, 1977

Niphargus Schiödte, 1847

Procrangonyx Schellenberg, 1934

Pseudocrangonyx Akatsuka et Komai, 1922

Super family Hadzioidea S. Karaman, 1943

Family MELITIDAE Bousfield, 1973

Melita Leach, 1814

Superfamily Gammaroidea Latreille, 1802

Family ACANTHOGAMMARIDAE Garjajew, 1901

Subfamily Acanthogammarinae Garjajew, 1901

Acanthogammarus Stebbing, 1899

$\mathrm{p}$

$\mathrm{p}$

$\mathrm{p}$

$\mathrm{p}$

$\mathrm{p}$

$\mathrm{m}$

Subfamily Garjaj ewiinae Tachteew, 2000 ${ }^{1}$

Family BEHNINGIELLIDAE Kamaltynov, 2001

Family CARINOGAMMARIDAE Tachteew, 2000

Family CASPICOLIDAE Birstein, 1945
Brandtia Bate, 1862

Burchania Tachteew, 2000

Carinurus Sowinsky, 1915

Coniurus Sowinsky, 1915

Propachygammarus Bazikalova, 1945

Cheirogammarus Sowinsky, 1915

Hyalellopsis Stebbing, 1899

Garjajewia Sowinsky, 1915

Koshovia Bazikalova, 1945

(6)

(1)

Paragarjajewia Bazikalova, 1945

(4)

Locustogammarus Bousfield, 1979

Spinulogammarus Tzvetkova, 1972

Behningiella Derzhavin, 1948

Cardiophilus G.O. Sars, 1896

Zernovia Derzhavin, 1948

Carinogammarus Stebbing, 1899

Echiuropus Sowinsky, 1915

Gmelinoides Bazikalova, 1945

Pseudomicruropus Bazikalova, 1962

$\mathrm{m}$

$\mathrm{m}$

$\mathrm{m}$

$\mathrm{m}$

$\mathrm{m}$

$\mathrm{m}$

$\mathrm{m}$

$\mathrm{m}$

$\mathrm{m}$

$\mathrm{m}$

$\mathrm{m}$

$\mathrm{m}$

Caspicola Derzhavin, 1945 $\mathrm{n}$

$\mathrm{n}$

n

$\mathrm{n}$

n

n

$\mathrm{n}$

m

$\mathrm{m}$ $\mathrm{m}(\mathrm{p} ?)$

$\mathrm{m}$

n 
Family GAMMARIDAE Lea ch, 1814

Abyssogammarus Sowinski, 1915

Amathillina Grimm in G.O. Sars, 1894

Axelboeckia Stebbing, 1899

Baku Karaman et Barnard, 1979

Bazikalovia Tachteew, 2000

Cephalogammarus Karaman et Barnard, 1979

Corophiomorphus Bazikalova, 1945

Derzhavinella Birstein, 1938

Echinogammarus Stebbing, $1899^{2}$

Eulimnogammarus Bazikalova, 1945

$\dagger$ Fluviogammarus Dorogostaisky, 1916

Gammarus J.C. Fabricius, 1775

Gmelina G.O. Sars, 1894

Gmelinopsis G.O. Sars, 1896

Heterogammarus Stebbing, 1899

Kuzmelina Karaman et Barnard, 1979

Lanceogammarus Karaman et Barnard, 1979

Lobogammarus Bazikalova, 1945

Macropereiopus Sowinsky, 1915

Odontogammarus Stebbing, 1899

Ommatogammarus Stebbing, 1899

Scytaelina Stock, Mirzajani, Vonk, Naderi et Kiabi, 1998

Shablogammarus Carausu, Dobreanu et Manolache, 1955

Sowinskya Derzhavin, 1948

Yogmelina Karaman et Barnard, 1979

Family IPHIGENELLIDAE Kama Itynov, 2001

Iphigenella G.O. Sars, 1896

Family MACROHECTOPODIDAE Sowinsky, 1915

Macrohectopus Stebbing, 1906

Family MICRUROPODIDAE Kamaltynov, 1999

Baikalogammarus Stebbing, 1899

Crypturopus Sowinsky, 1915

Homocerisca Bazikalova, 1945

Micruropus Stebbing, 1899

Micruropodidae (?) - incertae sedis

Profundalia Kamaltynov, 2001

Family PACHYSCHESIDAE Tachteew, 1998

Pachyschesis Bazikalova, 1945

Family PALLASEIDAE Tachteew, 2000 (1995 - nomen nudum)

Subfamily Pallaseinae Tachteew, 2000

Gymnogammarus Sowinsky, 1915

Hakonboeckia Stebbing, 1899

Leptostenus Bazikalova, 1945

Metapallasea Bazikalova, 1959

Pallasea Bate, 1862

Poekilogammarus Stebbing, 1899

Subfamily Parapallaseinae Kamaltynov, 1999 4

Ceratogammarus Sowinsky, 1915

Parapallase a Stebbing, 1899

Pallaseidae (?) - incertae sedis

Polyacanthisca Bazikalova, 1937
(5)

(2)

(5)

$\mathrm{m}$

$\mathrm{n}$

$\mathrm{n}$

n

n

$\mathrm{m}$

$\mathrm{n}$

m

$\mathrm{n}$

$\mathrm{n}(\mathrm{m} ?)$

$\mathrm{m}$

$\mathrm{m}$

m (p?)

$\mathrm{n}$

$\mathrm{n}$

$\mathrm{m}$

n

$\mathrm{n}$

$\mathrm{m}$

$\mathrm{m}$

$\mathrm{m}$

$\mathrm{m}$

n

n

n

$\mathrm{n}$

n

$\mathrm{m}$

(1)

(5)

(4)

(1)

(16) 
Family PONTOGAMMARIDAE Bousfield, 1977

Compactogammarus Stock, 1974

Dikerogammarus Stebbing, 1899

Niphargogammarus Birstein, 1945

Obesogammarus Stock, 1974

Pandorites G.O. Sars, 1895

Paraniphargoides Stock, 1974

Pontogammarus Sowinsky, 1904

$\mathrm{n}$

Superfamily Eusiro idea Bousfield, 1979

Family EUSIRIDAE Stebbing, 1888

Paramoera Miers, 1875

Sternomoera Barnard et Karaman, 1991

Family GAMMARACANTHIDAE Bousfield, 1989

Gammaracanthus Bate, 1862

Superfamily Lysia nassoidea Dana, 1849

Family URISTIDAE Hurley, 1963 Onisimus Boeck, 1871

Superfamily Phoxocephaloidea Sars, 1891

Family PONTOPOREIIDAE Dana, 1853

Pontoporeia Krøyer, 1842

(1) Monoporeia Bousfield, 1989

Superfamily Talitroidea Rafinesque, 1815

Family DOGIELINOTIDAE Gurjanova, 1953

Subfamily Dogielinotinae Gurjanova, 1953

Allorchestes Dana, 1849

(1) n

Dogielinotus Gurjanova, 1953

Family HY ALID AE Bulycheva, 1957

Subfamily Hya linae Bulycheva, 1957 Parallorchestes Shoemaker, 1941

(1) $\mathrm{n}$

Family TALITRIDAE Rafinesque, 1815

Orchestia Leach, 1814

Paciforchestia Bousfield, 1982

Platorchestia Bousfield, 1982

Traskorchestia Bousfield, 1982

Trinorchestia Bousfield, 1982

(2) n

(1) $\mathrm{n}$

(3) n

(2) n

(1) n

Suborder Corophiidea Leach, 1814

Superfamily Corophioidea Leach, 1814

Family COROPHIIDAE Leach, 1814

Subfamily Corophiinae Leach, 1814

Chelicorophium Bousfield et Hoover, 1997 


\author{
Corophium Latreille, 1806 \\ Monocorophium Bousfield et Hoover, 1997 \\ Family KAMAKIDAE Myers et Lowry, 2003 \\ Subfamily Kamakinae Myers et Lowry, 2003 \\ Kamaka Derzhavin, 1923
}

(1)

$\mathrm{n}$

$\mathrm{n}$

\footnotetext{
${ }^{1}$ Proposed as subfamily group in Takhteev [1997: 51], not named.

2 see section "Taxonomic notes".

${ }^{3}$ Preliminary diagnosis of this family was published in Takhteev [1998], but not in Kamaltynov [1999].

${ }^{4}$ Init ially proposed in Acanthogammari dae [Kamal tynov, 1999].
}

The ability of most amphipods to migrate long distances is a common behavioral trait and has facilitated their natural range expansion. According to the migration theory by Birstein [1935], the upstream migration of species in the rivers of the Sea of Azov and the Caspian and Black seas has resulted in the rapid dispersal of many amphipods from the south to the north of the former U.S.S.R. By the mid- $20^{\text {th }}$ century, the Ponto-Caspian amphipods Echinogammarus ischnus, Dikerogammarus haemobaphes, D. villosus and Obesogammarus obesus reached the middle part of the Volga River spreading upstream more than $4000 \mathrm{~km}$ from their native area [Berezina, 2007a].

In some cases, amphipods can be transferred to long distances as attached organisms by decapods, and birds [Rachalewski et al., 2013]. In other cases, large specimens of migrating amphipods were able to transport attached invertebrates (molluscs, rotifers and infusorians, etc.) being as a possible vector of accidental introductions for other species of invertebrates. For example, it is known that specimens of $D$. villosus have transported juveniles of the mollusc Dreissena spp. during migration upstream of the Ponto-Caspian rivers [Dedyu, 1963].

Below is a list of the species of allochthonous origin for Russian inland waters or so-called alien species. The recorded alien species were all placed in five complexes according to their origin.

1. Ponto-Caspian (PC) and the Mediterranean (Med.). The Ponto-Caspian complex includes species from the Caspian, Azov and Black seas, and from the watershed. The main vectors of their introduction to the Baltic Sea basin from southern latitudes are range extension across the Volga, Kama and Don rivers systems, due to the construction of artificial canals and reservoirs connecting different basins; transportation in ballast waters and the associated meta-cenosis of ships, and intentional introduction for fihery purposes. We included $O$. cavimana in this group considering the Meditterenian region to be its native area. It is also assumed, however, that according to the latest molecular data [Ketmaier, De Matthaeis, 2010], a so-called $O$. cavimana might be regarded as a species complex of a different origin.

2. Circumtropical (CT). This complex includes only one species Platorchestia platensis.
3. North American (NA). The North American complex includes the species that penetrated due to the development of the trans-Atlantic shipping.

4. Baikalian (B). The Baikalian complex includes species of Lake Baikal genesis, and species that are common for the Angara and Yenisei rivers and, more rarely, for the Ob' River. Their spread was primarily the result of the intentional introduction and a further range extension.

5. Local immigrants (Loc.). Local immigrants are species that penetrated Russia or its different regions from other European countries or other farther regions of Russia due to range extension. Gammarus lacustris Sars is one of the representative species in this group, although this species has a wide natural distribution in the European and Asian parts of Russia. It was introduced in Estonian, European Russian and Siberian lakes in order to increase the fish production or the abundance of native population. Attempts to acclimatize Gammarus lacustris from the Siberian population in Lake Pskovsko-Chudskoe were made during 1970-1975 [Timm, Timm, 1993]. In Eastern Siberia, fishermen used this species as a fish bait. They transfered a large number of $G$. lacustris adult specimens from one lake to another during a short fishing periods thus facilitating its successful re-introduction and local dispersal [Bekman, 1954].

The total list of alien species of European Russia includes 32 species. The largest number of species is represented by the species of the Ponto-Azov and the Caspian fauna complex (24 species) (Table 3).

\section{Results and Discussion}

The continental waters of Russia support 26 families, 110 genera and 581 species and sub-species of amphipods. Excluding species with uncertain taxonomic status, the Baikalian species account for $61 \%$ of fauna (276 species and 78 subspecies), the Ponto-Caspian species comprise $13 \%$ (78 species), the coastal brackish marine fauna $8 \%$ (47 species), and 6\% (35 species) are subterranean. Relicts of marine transgressions make up $2 \%$ (10 species), and terrestrial species less than $2 \%$ (9 species) [Takhteev, Sidorov, 2012]. The proportion of the species that expanded their range or even invaded the territory of Russia due to human activities constitutes $5.5 \%$ of the entire fauna (32 species). 
Table 2. Checklist.

Таблица 2. Контрольный список видов.

FAMILY ACANTHOGAMMARIDAE Garjajew, 1901 [12 Genera (G), 98 Species and subspe$\operatorname{cies}(\mathrm{S})]^{1}$

1. Acanthogammarus Stebbing, 1899

1. Acanthogammarus (Acanthogammarus) brevispinus Dorogostaisky, 1922 B

2. Acanthogammarus (Acanthogammarus) godlewskii (Dybowsky, 1874) B

- Gammarus godlewskii Dybowsky, 1874 (original combination) [designated by Dybowsky, 1924]

3. Acanthogammarus (Acanthogammarus) gracilispinus Tachteew, 2000 B

4. Acanthogammarus (Acanthogammarus) lappaceus lappaceus Tachteew, 2000 B

5. Acanthogammarus (Acanthogammarus) lappaceus longispinus Tachteew, 2000 B

6. Acanthogammarus (Acanthogammarus) maculosus Dorogostaisky, 1930 B

7. Acanthogammarus (Acanthogammarus) subbrevispinus Bazikalova, 1945 B

8. Acanthogammarus (Acanthogammarus) victorii (Dybowsky, 1874) B

9. Acanthogammarus (Brachyuropus) flavus flavus (Garjajew, 1901) B

10. Acanthogammarus (Brachyuropus) flavus curtus Bazikalova, 1945 B

11. Acanthogammarus (Brachyuropus) flavus rodionowi Dorogostaisky, 1922 B

12. Acanthogammarus (Brachyuropus) flavus sowinskii Bazikalova, 1945 B

13. Acanthogammarus (Brachyuropus) grewingkii (Dybowsky, 1874) B

14. Acanthogammarus (Brachyuropus) korotneffii (Garjajew, 1901) B

15. Acanthogammarus (Brachyuropus) nassonowi (Dorogostaisky, 1922) B

16. Acanthogammarus (Brachyuropus) reichertii (Dybowsky, 1874) B

2. Brandtia Bate, 1862

17. Brandtia (Brandtia) latissima acera (Dybowsky) in Dorogostaisky, $1916 \quad$ BE

18. Brandtia (Brandtia) latissima dicera (Dybowsky) in Dorogostaisky, $1916 \quad$ BE

19. Brandtia (Brandtia) latissima extima Dorogostaisky, 1930 B

20. Brandtia (Brandtia) latissima intermedia Dorogostaisky, $1930 \quad$ B

21. Brandtia (Brandtia) latissima lata (Dybowsky, 1874) B

22. Brandtia (Brandtia) latissima latior (Dybowsky, 1874) B

23. -Brandtia (Brandtia) latissima latissima (Gerstfeldt, 1858) [primary monotypy] B

Gammarus latissimus Gerstfeldt, 1858 (original combination)

24. Brandtia (Brandtia) latissima polyspina Dorogostaisky, 1930 B

25. Brandtia (Dorogostajskia) armata armata (Dybowsky, 1874) B

syn.: Dedyuola armata (Dybowsky, 1874), in: Kamaltynov [2001]

26. Brandtia (Dorogostajskia) armata ongureni (Garjajew, 1901) B

syn.: Dedyuola ongureni (Garjajew, 1901), in: Kamaltynov [2001]

27. Brandtia (Dorogostajskia) birsteini Bazikalova, 1948

28. Brandtia (Dorogostajskia) insularis (Dorogostaisky, 1930)

29. Brandtia (Dorogostajskia) margaritae Bazikalova, 1959

syn.: Dedyuola margaritae (Bazikalova, 1959), in: Kamaltynov [2001]

30. Brandtia (Dorogostaiskia) parasitica hanajevi (Daneliya et Väinölä, 2014) B

31. Brandtia (Dorogostaiskia) parasitica kamaltynovi (Daneliya et Väinölä, 2014) B

32. Brandtia (Dorogostajskia) parasitica parasitica (Dybowsky, 1874) B

33. Brandtia (Dorogostaiskia) parasitica stenocephala (Daneliya et Väinölä, 2014) B

34. Brandtia (Dorogostaiskia) parasitica ushkaniensis (Daneliya et Väinölä, 2014) B

3. Burchania Tachteew, 2000

35. Burchania meissneri (Bazikalova, 1935) [primary monotypy]

Hakonboeckia meissneri Bazikalova, 1935 (original combination)

4. Carinurus Sowinsky, 1915

36. Carinurus amentatus G. Karaman, 1976

37. Carinurus bazikalovae G. Karaman, 1976

B

38. Carinurus belkinii (Garjajew, 1901)

39. Carinurus bicarinatus Bazikalova, 1935

40. Carinurus bifrons G. Karaman, 1976

41. Carinurus microphthalmus (Sowinsky, 1915) 
42. Carinurus obscurus Dorogostaisky, 1922

43. Carinurus platycarinus (Sowinsky, 1915)

44. Carinurus reissnerii (Dybowsky, 1874)

45. Carinurus solskii (Dybowsky, 1874)

- Gammarus solskii Dybowsky, 1874 (original combination) [primary monotypy]

46. Carinurus werestschagini Bazikalova, 1935

5. Cheirogammarus Sowinsky, 1915

47. •Cheirogammarus inflatus Sowinsky, 1915 [primary monotypy]

6. Coniurus Sowinsky, 1915

48. • Coniurus palmatus Sowinsky, 1915 [designated by Bazikalova, 1945] B

49. Coniurus radoschkowskii (Dybowsky, 1874) B

50. Coniurus wadimi Sowinsky, 1915

7. Garjajewia Sowinsky, 1915

51. Garjajewia cabanisii cabanisii (Dybowsky, 1874)

- Gammarus cabanisii Dybowsky, 1874 (original combination) [designated by Bousfield, 1977]

52. Garjajewia cabanisii ninae Bazikalova, 1945

53. Garjajewia cabanisii pleshanovi Tachteew et Levashkevich, 2006

54. Garjajewia dershawini Sowinsky, 1915

55. Garjajewia dogieli Bazikalova, 1945

56. Garjajewia sarsi Sowinsky, 1915

8. Hyalellopsis Stebbing, 1899

57. Hyalellopsis (Boeckaxelia) carpenterii carpenterii (Dybowsky, 1874)

58. Hyalellopsis (Boeckaxelia) carpenterii elegans (Dorogostaisky, 1930)

59. Hyalellopsis (Boeckaxelia) carpenterii profundalis (Bazikalova, 1945)

60. Hyalellopsis (Boeckaxelia) potanini (Dorogostaisky, 1922)

61. Hyalellopsis (Boeckaxelia) rubra (Garjajew, 1901)

62. Hyalellopsis (Dorogammarus) castanea (Dorogostaisky, 1930)

63. Hyalellopsis (Gammarosphaera) insularis (Bazikalova, 1936)

64. Hyalellopsis (Hyalellopsis) bicolorata Bazikalova, 1948

65. Hyalellopsis (Hyalellopsis) carinata Sowinsky, 1915

66. Hyalellopsis (Hyalellopsis) costata Sowinsky, 1915

67. Hyalellopsis (Hyalellopsis) czyrnianskii (Dybowsky, 1874)

- Gammarus czyrnianskii Dybowsky, 1874 (original combination) [designated by Stebbing, 1899]

68. Hyalellopsis (Hyalellopsis) depressirostris Sowinsky, 1915

69. Hyalellopsis (Hyalellopsis) eugeniae Sowinsky, 1915

70. Hyalellopsis (Hyalellopsis) grisea Dorogostaisky, 1930

71. Hyalellopsis (Hyalellopsis) hamata Sowinsky, 1915

72. Hyalellopsis (Hyalellopsis) irinae Bazikalova, 1959

73. Hyalellopsis (Hyalellopsis) latipes latipes Bazikalova, 1945

74. Hyalellopsis (Hyalellopsis) latipes selengensis Bazikalova, 1945

75. Hyalellopsis (Hyalellopsis) macrocephala Bazikalova, 1945

76. Hyalellopsis (Hyalellopsis) nana Bazikalova, 1959

77. Hyalellopsis (Hyalellopsis) setosa Sowinsky, 1915

78. Hyalellopsis (Hyalellopsis) stebbingi Sowinsky, 1915

79. Hyalellopsis (Hyalellopsis) taczanowskii (Dybowsky, 1874)

80. Hyalellopsis (Hyalellopsis) tixtonae tixtonae Sowinsky, 1915

81. Hyalellopsis (Hyalellopsis) tixtonae glabra Bazikalova, 1945

82. Hyalellopsis (Hyalellopsis) variabilis Dorogostaisky, 1930

9. Koshovia Bazikalova, 1945

83. Koshovia mirabilis Bazikalova, 1975 [primary monotypy] 
85. Paragarjajewia petersii petersii (Dybowsky, 1874)

-Gammarus petersii Dybowsky, 1874 (original combination) [primary monotypy]

11. Plesiogammarus Stebbing, 1899

86. Plesiogammarus brevis brevis Bazikalova, 1975

87. Plesiogammarus brevis mazepowae Tachteew, 1997

88. Plesiogammarus brevis inquaesitus Tachteew, 1997

89. Plesiogammarus gerstaeckeri (Dybowsky, 1874)

-Gammarus gerstaeckeri Dybowsky, 1874 (original combination) [primary monotypy]

90. Plesiogammarus longicornis Sowinsky, 1915

91. Plesiogammarus martinsoni martinsoni Tachteew, 1997

92. Plesiogammarus martinsoni impransus Tachteew, 1997

93. Plesiogammarus timoshkini Tachteew, 1997

94. Plesiogammarus zienkowiczii (Dybowsky, 1874)

12. Propachygammarus Bazikalova, 1945

95. Propachygammarus bicornis (Dorogostaisky, 1930)

96. Propachygammarus dryshenkoi (Garjajew, 1901)

-Dybowskia dryshenkoi Garjajew, 1901 (original combination) [designated by Barnard, Barnard, 1983]

97. Propachygammarus lamellispinis (Bazikalova, 1945)

98. Propachygammarus maximus (Garjajew, 1901)

\section{FAMILY ANISOGAMMARIDAE Bousfield, 1977 [4 G 12 S]}

13. Eogammarus Birstein, 1933

99. Eogammarus barbatus (Tzvetkova, 1965)

100. Eogammarus itotomikoae Tomikawa, Morino, Toft et Mawatari, $2006 \quad$ SPC

101. Eogammarus kygi (Derzhavin, 1923)

-Gammarus kygi Derzhavin, 1923 (original combination) [designated by Tzvetkova, 1975]

102. Eogammarus possjeticus (Tzvetkova, 1967)

14. Jesogammarus Bousfield, 1979

105. Jesogammarus (Annanogammarus) annandalei (Tattersall, 1922)

- Gammarus annandalei Tattersall, 1922 (original combination) [designated by Bousfield, 1979]

15. Locustogammarus Bousfield, 1979

106. Locustogammarus aestuariorum (Tzvetkova, 1972)

107. Locustogammarus hirsutimanus (Kurenkov et Mednikov, 1959)

SPC

108. *Locustogammarus intermedius Labay, 1996

SPC

109. Locustogammarus locustoides (Brandt, 1851)

SPC

-Gammarus locustoides Brandt, 1851 (original combination) [designated by Bousfield, 1979]

16. Spinulogammarus Tzvetkova, 1972

110. Spinulogammarus ochotensis (Brandt, 1851)

-Gammarus ochotensis Brandt, 1851 (original combination) [designated by Tzvetkova, 1972]

2. FAMILY BEHNINGIELLIDAE Kamaltynov, $2001[3 \mathrm{G} 4 \mathrm{~S}]^{2}$

17. Behningiella Derzhavin, 1948

111. •Behningiella brachypus Derzhavin, 1948 
19. Zernovia Derzhavin, 1948

114. -Zernovia volgensis Derzhavin, 1948 [designated by Derzhavin, 1948]

PCE

3. FAMILY CARINOGAMMARIDAE Tachteew, 2000 [4G 29 S]

20. Carinogammarus Stebbing, 1899

115. Carinogammarus cinnamomeus (Dybowsky, 1874)

- Gammarus cinnamomeus Dybowsky, 1874 (original combination) [designated by Bazikalova, 1945]

116. Carinogammarus wagii wagii (Dybowsky, 1874)

B

117. Carinogammarus wagii pallidus (Dorogostaisky, 1922)

$\mathrm{B}$

21. Echiuropus Sowinsky, 1915

118. Echiuropus (Asprogammarus) bathyphilus (Bazikalova, 1975) B

119. Echiuropus (Asprogammarus) bekmanae Mekhanikova, Chapelle et De Broyer, 2001 B

120. Echiuropus (Asprogammarus) macropsis (Bazikalova, 1975) B

121. Echiuropus (Asprogammarus) puer (Bazikalova, 1975) B

122. Echiuropus (Asprogammarus) pulchelliformis (Bazikalova, 1975) B

123. Echiuropus (Asprogammarus) pulchellus (Dybowsky, 1874) B

124. Echiuropus (Asprogammarus) rhodophthalmus rhodophthalmus (Dybowsky, 1874) B

125. Echiuropus (Asprogammarus) rhodophthalmus brachyurus (Bazikalova, 1975) B

126. Echiuropus (Asprogammarus) rhodophthalmus microphthalmus (Dybowsky, 1874) B

127. Echiuropus (Asprogammarus) rhodophthalmus strenuus (Bazikalova, 1975) B

128. Echiuropus (Asprogammarus) seidlitzii (Dybowsky, 1874) B

129. Echiuropus (Echiuropus) levis Bazikalova, 1945

130. •Echiuropus (Echiuropus) macronychus macronychus Sowinsky, 1915 [primary monotypy] B

131. Echiuropus (Echiuropus) macronychus brevicaudatus Sowinsky, 1915 B

132. Echiuropus (Echiuropus) macronychus sempercarinatus (Bazikalova, 1975) B

133. Echiuropus (Echiuropus) morawitzii (Dybowsky, 1874) B

134. Echiuropus (Echiuropus) perplexus (Bazikalova, 1975) B

135. Echiuropus (Smaragdogammarus) gulekani (Bazikalova, 1975) B

136. Echiuropus (Smaragdogammarus) smaragdinus (Dybowsky, 1874) B

22. Gmelinoides Bazikalova, 1945

137. * Gmelinoides fasciatoides (Gurjanova, 1929) ${ }^{3} \quad$ BE (?)

138. Gmelinoides fasciatus (Stebbing, 1899)

- Brandtia fasciata Stebbing, 1899 [designated by Bousfield, 1977]

23. Pseudomicruropus Bazikalova, 1962

139. Pseudomicruropus chargoensis (Sowinsky, 1915) B

140. Pseudomicruropus lepidiformis Bazikalova, 1962 B

141. Pseudomicruropus lepidus (Bazikalova, 1945) B

142. Pseudomicruropus rotundatulus magnus Bazikalova, 1962 B

143. •Pseudomicruropus rotundatulus rotundatulus (Bazikalova, 1945) [designated by Bousfield, 1977]

Micruropus (Gammarisca) rotundatulus Bazikalova, 1945 (original combination)

4. FAMILY CASPICOLIDAE Birstein, 1945 [1 G 1 S $]$

24. Caspicola Birstein, 1945

144. Caspicola knipowitschi (Derzhavin, 1944)

PCE

- Caspiella knipowitschi Derzhavin, 1944 (original combination) [primary monotypy]

5. FAMILY COROPHIIDAE Leach, 1814 [3 G $12 \mathrm{~S}]$

25. Chelicorophium Bousfield et Hoover, 1997

145. Chelicorophium chelicorne (G.O. Sars, 1895) 
146. Chelicorophium curvispinum (G.O. Sars, 1895)

PCE

- Corophium curvispinum G.O. Sars, 1895 (original combination) [designated by Bousfield, Hoover, 1997]

147. Chelicorophium maeoticum (Sowinsky, 1898)

PCE

148. Chelicorophium monodon (G.O. Sars, 1895)

PCE

149. Chelicorophium mucronatum (G.O. Sars, 1895)

PCE

150. Chelicorophium nobile (G.O. Sars, 1895)

PCE

151. Chelicorophium robustum (G.O. Sars, 1895)

PCE

152. Chelicorophium sowinskyi (Martynov, 1924)

PCE

153. Chelicorophium spinulosum (G.O. Sars, 1896)

PCE

154. *Chelicorophium spongicolum (Welitchkovsky, 1914) ${ }^{4}$

PCE

26. Corophium Latreille, 1806

Type species: Oniscus volutator Pallas, 1766, primary monotypy.

155. Corophium volutator (Pallas, 1766)

156. Corophium orientale Schellenberg, 1928

PCE, BSE

27. Monocorophium Bousfield et Hoover, 1997

Type species: Corophium insidiosum Crawford, 1937, designated by Bousfield \& Hoover [1997].

157. Monocorophium steinegeri (Gurjanova, 1951)

SPC

6. FAMILY CRANGONYCTIDAE Bousfield, 1973 [4 G 12 S]

28. Amurocrangonyx Sidorov et Holsinger, 2007

158. Amurocrangonyx arsenjevi (Derzhavin, 1927)

SPC

-Eucrangonyx arsenjevi Derzhavin, 1927 (original combination) [primary monotypy]

29. Crangonyx Bate, 1859

Type species: Crangonyx subterraneus Bate, 1859, primary monotypy.

159. Crangonyx chlebnikovi Borutzky, 1928

WP

30. Lyurella Derzhavin, 1939

Type species: Lyurella hyrcana Derzhavin, 1939, primary monotypy.

160. Lyurella shepsiensis Sidorov, 2015

31. Stygobromus Cope, 1872

Type species: Stygobromus vitreus Cope, 1872, primary monotypy.

161. Stygobromus anastasiae Sidorov, Holsinger et Takhteev, 2010

CA

162. Stygobromus mikhaili Sidorov, Holsinger et Takhteev, 2010

CA

163. Stygobromus pusillus (Martynov, 1930)

32. Synurella Wrześniowski, 1877

164. Synurella ambulans (F. Müller, 1846)

-Gammarus ambulans F. Müller, 1846 (original combination) [designated by Wrześniowski, 1877] syn.: Synurella meschtscherica Borutzky, 1929

165. Synurella behningi Birstein, 1948

166. Synurella derzhavini Behning, 1928

167. Synurella donensis Martynov, 1919

WP

168. Synurella jakutana Martynov, 1931

WP

169. Synurella levanidovae G. Karaman, 1991

SPC

170. Synurella stadukhini Derzhavin, 1930

SPC

SPC 
FAMILY DOGIELINOTIDAE Gurjanova, 1953 [2 G 2 S ]

33. Allorchestes Dana, 1849

Type species: Allorchestes compressa Dana, 1852, designated by Chevreux \& Fage [1925].

171. Allorchestes malleola Stebbing, 1899

SPC

syn.: Allorchestes vladimiri Derzhavin, 1937

34. Dogielinotus Gurjanova, 1953

172. Dogielinotus moskvitini (Derzhavin, 1930)

-Allorchestes moskvitini Derzhavin, 1930 (original combination) [designated by Gurjanova, 1953]

7. FAMILY EUSIRIDAE Stebbing, 1888 [2 G 5 S]

35. Paramoera Miers, 1875

Type species: Paramoera australis Miers, 1875, designated by Miers [1875].

173. Paramoera anivae Labay, 2012

SPC

174. Paramoera (Ganigamoera) myslenkovi Sidorov, 2010

$\mathrm{SPC}$

175. Paramoera (Ganigamoera) tiunovi Sidorov, 2010

$\mathrm{SPC}$

176. *Paramoera (Paramoera) udehe (Derzhavin, 1930)

SPC

36. Sternomoera Barnard et Karaman, 1991

177. Sternomoera moneronensis Labay, 1997

$\mathrm{SPC}$

178. Sternomoera yezoensis (Uéno, 1933)

$\mathrm{SPC}$

-Paramoera yezoensis Uéno, 1933 (original combination) [designated by Barnard, Karaman, 1991]

8. FAMILY GAMMARACANTHIDAE Bousfield, 1989 [1 G 3 (4?) S]

37. Gammaracanthus Bate, 1862

Type species: Gammarus loricatus Sabine, 1821, designated by Bate [1862].

179. Gammaracanthus loricatus aestuariorum Lomakina in Gurjanova, 1951

AOE

syn.: Gammaracanthus loricatus ostiorum Lomakina in Gurjanova, 1951

180. Gammaracanthus loricatus caspius (G.O. Sars, 1896)

AOE (?)

181. Gammaracanthus lacustris Sars, 1867

AOE

syn.: Gammaracanthus relictus G.O. Sars, 1895

syn.: Relictacanthus lacustris (G.O. Sars, 1867)

182. *Gammaracanthus loricatus baicalensis Sowinski, $1915^{5}$

$\mathrm{AOE}(?)$

9. FAMILY GAMMARIDAE Leach, 1814 [26 G $171 \mathrm{~S}]$

38. Abyssogammarus Sowinski, 1915

183. Abyssogammarus gracilis gracilis Sowinski, 1915

184. Abyssogammarus gracilis minor Bazikalova, 1945

B

185. •Abyssogammarus sarmatus sarmatus (Dybowsky, 1874) [designated by Bazikalova, 1945]

186. Abyssogammarus sarmatus echinatus Bazikalova, 1935

187. Abyssogammarus swartschewskii Sowinski, 1915

39. Akerogammarus Derzhavin et Pjatakova, 1967

188. Akerogammarus contiguus (Pjatakova, 1962) 
191. • Amathillina cristata Grimm in G.O. Sars, 1894 [designated by G.O. Sars, 1894] PCE

192. Amathillina maximovitschi G.O. Sars, 1896

193. Amathillina pusilla G.O. Sars, 1896

PCE

194. Amathillina spinosa Grimm in G.O. Sars, 1896

PCE

PCE

41. Axelboeckia Stebbing, 1899

195. Axelboeckia spinosa (Grimm in G.O. Sars, 1894)

PCE

-Boeckia spinosa Grimm in G.O. Sars, 1894 (original combination) [primary monotypy; by G.O. Sars, 1894]

42. Baku Karaman et Barnard, 1979

196. Baku paradoxus (Derzhavin in Derzhavin et Pjatakova, 1967)

PCE

-Pontogammarus paradoxus Derzhavin, 1967 (original combination) [primary monotypy]

43. Bazikalovia Tachteew, 2000

197. Bazikalovia minima (Bazikalova, 1945) B

198. Bazikalovia obsoleta (Bazikalova, 1945) B

199. Bazikalovia simplex (Sowinsky, 1915) B

-Microgammarus simplex Sowinsky, 1915 (original combination) [designated by Takhteev, 2000]

200. Bazikalovia simpliciformis (Bazikalova, 1975)

44. Cephalogammarus Karaman et Barnard, 1979

201. Cephalogammarus macrocephalus (G.O. Sars, 1896)

PCE

- Gammarus macrocephalus G.O. Sars, 1896 (original combination) [designated by Karaman, Barnard, 1979]

45. Corophiomorphus Bazikalova, 1945

202. Corophiomorphus calceolatus (Sowinsky, 1915) B

203. Corophiomorphus crassicornis (Sowinsky, 1915) B

204. Corophiomorphus gracilicornis (Bazikalova, 1945) B

205. Corophiomorphus kietlinskii (Dybowsky, 1874) B B

206. Corophiomorphus laevis (Sowinsky, 1915) B

207. Corophiomorphus macrophthalmus (Bazikalova, 1945) B

208. Corophiomorphus pachycerus (Bazikalova, 1945) B

209. Corophiomorphus sophiae (Dybowsky, 1874) B

-Gammarus sophiae Dybowsky, 1874 (original combination) [designated by Barnard, Barnard, 1983]

210. Corophiomorphus stanislavii (Dybowsky, 1874)

211. Corophiomorphus tenuipes (Sowinsky, 1915)

46. Derzhavinella Birstein, 1938

212. Derzhavinella cava Stock, Mirzajani, Vonk, Naderi et Kiabi, 1998

213. •Derzhavinella macrochelata Birstein, 1938 [primary monotypy]

47. Echinogammarus Stebbing, 1899

Type species: Gammarus berilloni Catta, 1878, selected by Chevreux \& Fage [1925]

214. Echinogammarus foxi (Schellenberg, 1928)

PC

215. Echinogammarus ischnus (Stebbing, 1899)

PCE

syn.: Gammarus tenellus Sars, 1896

syn.: Gammarus sowinskyi Behning, 1914

syn.: Chaetogammarus tenellus behningi Martynov, 1919

syn.: Chaetogammarus ischnus (Stebbing, 1899)

216. *Echinogammarus placidus (Grimm in G.O. Sars, 1896)

217. Echinogammarus trichiatus (Martynov, 1932)

syn.: Chaetogammarus tenellus major Cărăuşu, 1943 
48. Eulimnogammarus Bazikalova, $1945^{6}$

219. Eulimnogammarus (Eulimnogammarus) burkani Bazikalova, 1945

220. Eulimnogammarus (Eulimnogammarus) cruentus (Dorogostaisky, 1930) B

221. Eulimnogammarus (Eulimnogammarus) cyanoides (Sowinsky, 1915) B

222. Eulimnogammarus (Eulimnogammarus) czerskii (Dybowsky, 1874) B

223. Eulimnogammarus (Eulimnogammarus) grandimanus Bazikalova, 1945 B

224. Eulimnogammarus (Eulimnogammarus) heterochirus Bazikalova, 1945 B

225. Eulimnogammarus (Eulimnogammarus) lividus lividus (Dybowsky, 1874) B

226. Eulimnogammarus (Eulimnogammarus) lividus angarensis (Dorogostaisky, 1916) BE

227. Eulimnogammarus (Eulimnogammarus) maackii maackii (Gerstfeldt, 1858) B

228. Eulimnogammarus (Eulimnogammarus) maackii brevicauda Bazikalova, $1957 \quad$ BE

229. Eulimnogammarus (Eulimnogammarus) macrochirus (Bazikalova, 1945) B

230. Eulimnogammarus (Eulimnogammarus) messerschmidtii Bedulina et Tachteew, 2014 B

231. Eulimnogammarus (Eulimnogammarus) verrucosus verrucosus (Gerstfeldt, 1858) [designated by Bazikalova, 1945]

- Gammarus verrucosus Gerstfeldt, 1858 (original combination)

232. Eulimnogammarus (Eulimnogammarus) verrucosus oligacanthus Bazikalova, $1945 \quad$ B

233. Eulimnogammarus (Eulimnogammarus) viridiformis (Sowinsky, 1915) B

234. Eulimnogammarus (Eurybiogammarus) affinis (Sowinsky, 1915) B

235. Eulimnogammarus (Eurybiogammarus) aheneoides Bazikalova, 1945 B

236. Eulimnogammarus (Eurybiogammarus) aheneus aheneus (Dybowsky, 1874) B

237. Eulimnogammarus (Eurybiogammarus) aheneus asetus Bazikalova, 1945 B

238. Eulimnogammarus (Eurybiogammarus) aheneus setosus (Dybowsky, 1874) B

239. Eulimnogammarus (Eurybiogammarus) brachycoxalis Bazikalova, 1945 B

240. Eulimnogammarus (Eurybiogammarus) byrkini (Sowinsky, 1915) B

241. Eulimnogammarus (Eurybiogammarus) capreolus (Dybowsky, 1874) B

242. Eulimnogammarus (Eurybiogammarus) epimeralis (Sowinsky, 1915) B

243. Eulimnogammarus (Eurybiogammarus) fuscus fuscus (Dybowsky, 1874) B

244. Eulimnogammarus (Eurybiogammarus) fuscus aureus (Dorogostaisky, $1916 \quad$ BE

245. Eulimnogammarus (Eurybiogammarus) fuscus longicornis Bazikalova, 1945 B

246. Eulimnogammarus (Eurybiogammarus) ibex ibex (Dybowsky, 1874) B

247. Eulimnogammarus (Eurybiogammarus) ibex atrichus Bazikalova, 1945 B

248. Eulimnogammarus (Eurybiogammarus) kusnezowi (Sowinsky, 1915) B

249. Eulimnogammarus (Eurybiogammarus) muriniformis Bazikalova, 1945 B

250. Eulimnogammarus (Eurybiogammarus) murinus (Dybowsky, 1874) B

251. Eulimnogammarus (Eurybiogammarus) parvexiformis Bazikalova, 1945 B

252. Eulimnogammarus (Eurybiogammarus) parvexii (Dybowsky, 1874) B

253. Eulimnogammarus (Eurybiogammarus) polyarthrus (Dybowsky, 1874) B

254. Eulimnogammarus (Eurybiogammarus) proximus (Sowinsky, 1915) B

255. Eulimnogammarus (Eurybiogammarus) rachmanowi (Sowinsky, 1915) B

256. Eulimnogammarus (Eurybiogammarus) saphirinus (Dybowsky, 1874) B

257. Eulimnogammarus (Eurybiogammarus) schamanensis (Dybowsky, 1874) B

258. Eulimnogammarus (Eurybiogammarus) similis (Sowinsky, 1915) B

259. Eulimnogammarus (Eurybiogammarus) stenophthalmus (Dybowsky, 1874) B

260. Eulimnogammarus (Eurybiogammarus) toxophthalmus (Dybowsky, 1874) B

261. Eulimnogammarus (Eurybiogammarus) ussolzewii ussolzewii (Dybowsky, 1874) B

262. Eulimnogammarus (Eurybiogammarus) ussolzewii abyssorum (Dybowsky, 1874) B

263. Eulimnogammarus (Eurybiogammarus) violaceus (Dybowsky, 1874) B

264. Eulimnogammarus (Eurybiogammarus) virgatus (Dorogostaisky, 1930) B

265. Eulimnogammarus (Philolimnogammarus) cyanellus Bazikalova, 1945 B

266. Eulimnogammarus (Philolimnogammarus) cyaneus cyaneus (Dybowsky, 1874) B

267. Eulimnogammarus (Philolimnogammarus) cyaneus angarensis Bazikalova, $1957 \quad$ BE

268. Eulimnogammarus (Philolimnogammarus) cyaneus comatus (Dorogostaisky, 1916) BE

269. Eulimnogammarus (Philolimnogammarus) exiguus Bazikalova, 1945 
Table 2 (contituing) Таблица 2 (продолжение)

270. Eulimnogammarus (Philolimnogammarus) immundus Bazikalova, 1945

271. Eulimnogammarus (Philolimnogammarus) inconspicuus Bazikalova, 1945

272. Eulimnogammarus (Philolimnogammarus) marituji Bazikalova, 1945

273. Eulimnogammarus (Philolimnogammarus) melanochlorus (Dorogostaisky, 1930)

274. Eulimnogammarus (Philolimnogammarus) testaceus (Dybowsky, 1874)

275. Eulimnogammarus (Philolimnogammarus) viridis viridis (Dybowsky, 1874)

276. Eulimnogammarus (Philolimnogammarus) viridis canus (Dybowsky, 1874)

277. Eulimnogammarus (Philolimnogammarus) viridis olivaceus (Dybowsky, 1874)

278. Eulimnogammarus (Philolimnogammarus) viridulus Bazikalova, 1945

279. Eulimnogammarus (Philolimnogammarus) vittatus (Dybowsky, 1874)

49. †Fluviogammarus Dorogostaisky, $1916^{7}$

280. $\uparrow$ Fluviogammarus angarensis Bazikalova, 1945

$\mathrm{BE}$

281. $\uparrow$ Fluviogammarus brachyurus (Dorogostaisky, 1916)

$\mathrm{BE}$

282. † Fluviogammarus intermedius Bazikalova, 1945

$\mathrm{BE}$

283. • Fluviogammarus larviformis Dorogostaisky, 1916 [primary monotypy]

$\mathrm{BE}$

50. Gammarus J.C. Fabricius, 1775

balcanicus species group

284. Gammarus balcanicus Schäferna, 1922

syn.: Gammarus angustatus Martynov, 1930

285. Gammarus suifunensis Martynov, 1925

WP, CA

SPC

locusta species group

286. Gammarus duebeni Liljeborg, 1852

WP

287. Gammarus inaequicauda Stock, 1996

WP

288. Gammarus kamtschaticus Tzvetkova, 1972

SPC

289. Gammarus locusta (Linnaeus, 1758)

290. Gammarus oceanicus Segerstråle, 1947

WP

291. Gammarus setosus Dementieva, 1931

WP

292. Gammarus spooneri G. Karaman, 1991

WP

syn.: Gammarus salinus Spooner, 1947

293. Gammarus wilkitzkii Birula, 1897

WP

294. Gammarus zaddachi Sexton, 1912

SPC

WP

pulex species group

295. Gammarus aequicauda (Martynov, 1931)

PCE

296. *Gammarus angulatus (Martynov, 1930)

CA

297. *Gammarus barnaulensis Schellenberg, $1937^{8}$

CA (?)

298. *Gammarus caucasicus Martynov, 1932

299. *Gammarus chostensis Martynov, 1932

$\tilde{\mathrm{N}}$

300. *Gammarus crispus Martynov, 1932

301. Gammarus dabanus Tachteew et Mekhanikova, 2000

302. Gammarus jacksoni Morino et Whitman, 1995

SPC

303. Gammarus komareki Schäferna, 1922

WP

304. Gammarus korbuensis Martynov, 1930

CA

305. Gammarus koreanus Uéno, 1940

SPC

306. Gammarus lacustris Sars, 1863

$\mathrm{H}$

syn.: Gammarus pulex extensus Martynov, 1931

syn.: Gammarus pulex karae Birula, 1937

syn.: Gammarus lacustris krokurensis Kurenkov et Mednikov, 1959

307. Gammarus pellucidus Gurjanova, 1929

WP, SPC (?)

308. Gammarus pulex (Linnaeus, 1758) ${ }^{9}$

- Cancer pulex Linnaeus, 1758 (original combination) [designated by Latreille, 1810]

309. Gammarus teletzkensis Martynov, 1930

310. Gammarus tigrinus Sexton, 1939 
51. Gmelina G.O. Sars, 1894

311. Gmelina aestuarica (Carausu, 1943)

PCE

312. •Gmelina costata G.O. Sars, 1894 [designated by G.O. Sars, 1894]

PCE

52. Gmelinopsis G.O. Sars, 1896

313. Gmelinopsis aurita G.O. Sars, 1896

PCE

314. •Gmelinopsis tuberculata G.O. Sars, 1896 [designated by G.O. Sars, 1896]

PCE

53. Heterogammarus Stebbing, 1899

315. Heterogammarus capellus capellus (Dybowsky, 1874)

316. Heterogammarus capellus sowinskii (Bazikalova, 1945)

317. Heterogammarus sophianosii (Dybowsky, 1874)

-Gammarus sophianosii Dybowsky, 1874 (original combination) [designated by Stock, 1969]

54. Kuzmelina Karaman et Barnard, 1979

318. Kuzmelina kusnezowi (Sowinsky, 1894)

- Gmelina kusnezowi Sowinsky, 1894 (original combination) [designated by Karaman, Barnard, 1979]

55. Lanceogammarus Karaman et Barnard, 1979

319. Lanceogammarus andrussovi (G.O. Sars, 1896)

PCE

- Gammarus andrussowi G.O. Sars, 1896 (original combination) [designated by Karaman, Barnard, 1979]

56. Lobogammarus Bazikalova, 1945

320. •Lobogammarus latus Bazikalova, 1945 [primary monotypy]

57. Macropereiopus Sowinsky, 1915

321. Macropereiopus albulus (Dybowsky, 1874)

322. Macropereiopus florii (Dybowsky, 1874)

- Gammarus florii Dybowsky, 1874 (original combination) [designated by Dybowski, 1924]

323. Macropereiopus grandimanus Bazikalova, 1975

324. Macropereiopus leucophthalmus (Sowinsky, 1915)

325. Macropereiopus mirus Bazikalova, 1975

326. Macropereiopus parvus Bazikalova, 1945

327. Macropereiopus wagneri wagneri Sowinsky, 1915

328. Macropereiopus wagneri dagarskii Sowinsky, 1915

$\mathrm{B}$

58. Odontogammarus Stebbing, 1899

329. Odontogammarus bekmanae Tachteew, 1999

330. Odontogammarus brevipes Dorogostaisky, 1930

331. Odontogammarus calcaratus calcaratus (Dybowsky, 1874)

- Gammarus calcaratus Dybowsky, 1874 (original combination) [designated by Dybowski, 1924]

332. Odontogammarus calcaratus caeculus Tachteew, 1999

333. Odontogammarus calcaratus improvisus Dorogostaisky, 1930

334. Odontogammarus calcaratus pulcherrimus Dorogostaisky, 1930

335. Odontogammarus demianowiczi Dorogostaisky, 1930 B

336. Odontogammarus korotnewi (Sowinsky, 1915)

337. Odontogammarus margaritaceus (Dybowsky, 1874)

59. Ommatogammarus Stebbing, 1899

338. Ommatogammarus albinus (Dybowsky, 1874)

339. Ommatogammarus carneolus carneolus (Dybowsky, 1874)

340. Ommatogammarus carneolus amethystinus (Dybowsky, 1874)

341. Ommatogammarus carneolus melanophthalmus Dorogostaisky in Bazikalova, 1945

342. Ommatogammarus flavus (Dybowsky, 1874) ${ }^{10}$

- Gammarus flavus Dybowsky, 1874 (original combination) [designated by Dybowski, 1924]

343. Ommatogammarus hyacinthinus (Dybowsky, 1874) 
60. Scytaelina Stock, Mirzajani, Vonk, Naderi et Kiabi, 1998

61. Shablogammarus Carausu, Dobreanu et Manolache, 1955

345. Shablogammarus chablensis (Carausu, 1943)

- Gammarus chablensis Carausu, 1943 (original combination) [primary monotypy]

346. Shablogammarus subnudus (G.O. Sars, 1896)

62. Sowinskya Derzhavin, 1948

347. • Sowinskya macrocera Derzhavin, 1948 [primary monotypy] PCE

63. Yogmelina Karaman et Barnard, 1979

348. Yogmelina brachyura (Derzhavin et Pjatakova, 1967)

349. Yogmelina cocolita G.S. Karaman et J.L. Barnard, 1979

PCE

syn.: Yogmelina pusilla Carausu, 1943

350. Yogmelina laeviuscula (G.O. Sars, 1896)

351. Yogmelina limana G.S. Karaman et J.L. Barnard, 1979

PCE

352. Yogmelina ovata (Martynov, 1924)

353. Yogmelina pusilla (G.O. Sars, 1896)

PCE

- Gmelina pusilla G.O. Sars, 1896 (original combination) [designated by Karaman, Barnard, 1979]

10. FAMILY HYALIDAE Bulycheva, 1957 [2 G 2 S]

64. Parallorchestes Shoemaker, 1941

354. Parallorchestes ochotensis (Brandt, 1851)

SPC

-Allorchestes ochotensis Brandt, 1851 (original combination) [designated by Shoemaker, 1941]

syn.: Parhyale kurilensis Iwasa, 1934

11. FAMILY IPHIGENELLIDAE Kamaltynov, $2001\left[\begin{array}{lll}1 & \mathrm{G} & 1\end{array}\right.$

65. Iphigenella G.O. Sars, 1896

355. •Iphigenella acanthopoda G.O. Sars, 1896 [primary monotypy]

12. FAMILY KAMAKIDAE Myers et Lowry, 2003 [ [ 1 G 1 S]

66. Kamaka Derzhavin, 1923

356. •Kamaka kuthae Derzhavin, 1923 [primary monotypy]

13. FAMILY MACROHECTOPODIDAE Sowinsky, 1915 [1 G 1 S]

67. Macrohectopus Stebbing, 1906

357. Macrohectopus branickii (Dybowsky, 1874)

-Constantia branickii Dybowsky, 1874 (original combination) [primary monotypy]

14. FAMILY MELITIDAE Bousfield, 1973 [1 G 1 S]

68. Melita Leach, 1814

Type species: Cancer palmata Montagu, 1804, primary monotypy.

358. Melita nitidaformis Labay, 2003

15. FAMILY MICRUROPODIDAE Kamaltynov, 1999 [4G 52 S]

69. Baikalogammarus Stebbing, 1899

359. Baikalogammarus pullus (Dybowsky, 1874)

-Gammarus pullus Dybowsky, 1874 (original combination) [primary monotypy; by Stebbing, 1899]

70. Crypturopus Sowinsky, 1915

360. Crypturopus inflatus (Dybowsky, 1874) 
-Gammarus inflatus Dybowsky, 1874 (original combination) [designated by Dybowski, 1924]

361. Crypturopus pachytus (Dybowsky, 1874)

362. Crypturopus rugosus (Dybowsky, 1874)

363. Crypturopus tenuipes Bazikalova, 1945

364. Crypturopus tuberculatus (Dybowsky, 1874)

71. Homocerisca Bazikalova, 1945

365. Homocerisca caudata Bazikalova, 1945

366. Homocerisca perla (Dybowsky, 1874)

- Gammarus perla Dybowsky, 1874 (original combination) [designated by Barnard, Barnard, 1983]

367. Homocerisca perloides Bazikalova, 1945

368. Homocerisca tenuicauda Bazikalova, 1975

72. Micruropus Stebbing, 1899

369. Micruropus asper Bazikalova, 1962

370. Micruropus brevicauda Bazikalova, 1945

B

7icruropus ciliodorsalis ciliodorsalis Sowinsky, 1915

372. Micruropus ciliodorsalis rostratus Bazikalova, $1962 \quad$ B

373. Micruropus cristatus Dorogostaisky, 1936 B

374. Micruropus dybowskii Bazikalova, 1945 B

375. Micruropus eugenii Bazikalova, 1959 B

376. Micruropus fixsenii (Dybowsky, 1874) B

-Gammarus fixsenii Dybowsky, 1874 (original combination) [designated by Dybowski, 1924; the later designation [Bazikalova, 1945] of Gammarus wohlii Dybowsky, 1874 is invalid]

377. Micruropus galasii Bazikalova, 1962

378. Micruropus glaber glaber (Dybowsky, 1874)

379. Micruropus glaber murini Bazikalova, 1945

380. Micruropus ivanowi ivanowi Bazikalova, 1945 B

381. Micruropus ivanowi garjajewi Bazikalova, 1945

382. Micruropus klukii (Dybowsky, 1874) B

383. Micruropus koshowi crassicauda Bazikalova, 1962 B

384. Micruropus koshowi koshowi Bazikalova, 1945

385. Micruropus koshowi setosus Bazikalova, 1945 B

386. Micruropus laeviusculus dubius Bazikalova, 1945

387. Micruropus laeviusculus laeviusculus (Sowinsky, 1915) B

388. Micruropus littoralis crassipes Sowinsky, 1915 B

389. Micruropus littoralis littoralis (Dybowsky, 1874) B B

390. Micruropus macroconus calceolaris Bazikalova, $1945 \quad$ B

391. Micruropus macroconus gurjanowae Bazikalova, 1945 B

392. Micruropus macroconus macroconus Bazikalova, $1945 \quad$ B

393. Micruropus macroconus tenuis Bazikalova, $1945 \quad$ B

394. Micruropus minutus (Sowinsky, 1915) B

395. Micruropus mozi Bazikalova, 1945 B

396. Micruropus parvulus Bazikalova, 1945 B

397. Micruropus possolskii Sowinsky, 1915 B

398. Micruropus pupilla Bazikalova, 1962 B

399. Micruropus pusillus Bazikalova, 1962 B

400. Micruropus semenowi Bazikalova, 1945 B

401. Micruropus talitroides angarensis Dorogostaisky, $1916 \quad$ BE

402. Micruropus talitroides eurypus Bazikalova, $1945 \quad$ B

403. Micruropus talitroides latus Bazikalova, 1962 B

404. Micruropus talitroides talitroides (Dybowsky, 1874) B

405. Micruropus ushkani Bazikalova, 1945

406. Micruropus vortex angarensis Bazikalova, $1962 \quad$ BE

407. Micruropus vortex vortex (Dybowsky, 1874) B

408. Micruropus vortex vorticellus Bazikalova, 1945

409. Micruropus wohlii platycercus (Dybowsky, 1874) B B

410. Micruropus wohlii wohlii (Dybowsky, 1874) B B 
16. FAMILY NIPHARGIDAE Bousfield, 1977 [1 G 9 S]

73. Niphargus Schiödte, 1847

Type species: Niphargus stygius Schiödte, 1849, primary monotypy.

elegans-valachicus species group ${ }^{11}$

411. Niphargus cubanicus Birstein, 1954

412. Niphargus potamophilus Birstein, 1954

skopljensis species group

413. *Niphargus submersus (Derzhavin, 1945)

stygius-puteanus species group

414. Niphargus abchasicus Martynov, 1932

C

415. Niphargus caelestis G.S. Karaman, 1982

C

syn.: Niphargus stygius longidactylus Birstein, 1952

416. Niphargus krasnodarus Karaman, 2012

C

417. Niphargus latimanus (Birstein, 1952)

syn.: Niphargus stygius latimanus Birstein, 1952

418. Niphargus pseudolatimanus (Birstein, 1952)

syn.: Niphargus stygius pseudolatimanus Birstein, 1952

tatrensis species group

419. Niphargus smirnovi Birstein, 1952

17. FAMILY OEDICEROTIDAE Lilljeborg, 1865 [3 G 3 S]

74. Acanthostepheia Boeck, 1871

Type species: Amphithonotus malmgreni Goes, 1866, primary monotypy.

420. Acanthostepheia incarinata Gurjanova, 1929

AOE

75. Deflexilodes Bousfield et Chevrier, 1996

Type species: Monoculodes tenuirostratus Boeck, 1871, designated by Bousfield, Chevrier [1996].

421. Deflexilodes minutus (Gurjanova, 1929)

AOE

76. Oediceros Krøyer, 1842

Type species: Oediceros saginatus Krøyer, 1842, primary monotypy.

422. Oediceros minor Gurjanova, 1930

AOE

18. FAMILY PACHYSCHESIDAE Tachteew, 1998 [1 G 16 S]

77. Pachyschesis Bazikalova, 1945

423. Pachyschesis acanthogammarii Tachteew, 2000 B

424. Pachyschesis bazikalovae G. Karaman, 1976 B

425. Pachyschesis bergi Bazikalova, 1945 B

426. Pachyschesis branchialis (Dybowsky, 1874) B

- Gammarus branchialis Dybowsky, 1874 (original combination) [designated by Barnard, Barnard, 1983]

427. Pachyschesis bumammus Tachteew, 2000 B

428. Pachyschesis crassus (Sowinsky, 1915) B

429. Pachyschesis cucuschonok Tachteew, 2000 B

430. Pachyschesis indiscretus Tachteew, 2000 B

431. Pachyschesis inquilinus Tachteew, 2000 B

432. Pachyschesis karabanowi Tachteew, 2000 B 
433. Pachyschesis lamakini Tachteew, 2000

B

434. Pachyschesis pinguiculus Tachteew, 2000

B

435. Pachyschesis punctiommatus Tachteew, 2000

436. Pachyschesis rarus Tachteew, 2000

437. Pachyschesis sideljowae Tachteew, 2000

B

438. Pachyschesis vorax Tachteew, 2000

19. FAMILY PALLASEIDAE Tachteew, 2000 (Tachteev 1995a, nomen nudum) [8 G 57 S]

78. Ceratogammarus Sowinsky, 1915

439. Ceratogammarus acerus Bazikalova, 1937 B

440. Ceratogammarus cornutus (Sowinsky, 1915) B

441. • Ceratogammarus dybowskii Sowinsky, 1915 [primary monotypy] [designated by Barnard, Barnard, 1983]

79. Gymnogammarus Sowinsky, 1915

442. •Gymnogammarus macrurus Sowinsky, 1915 [primary monotypy]

80. Hakonboeckia Stebbing, 1899

443. •Hakonboeckia strauchii (Dybowsky, 1874) [primary monotypy; by Stebbing, 1899] B

81. Leptostenus Bazikalova, 1945

444. •Leptostenus leptocerus (Dybowsky, 1874) [primary monotypy]

82. Metapallasea Bazikalova, 1959

445. $\bullet$ Metapallasea galinae Bazikalova, 1959 [primary monotypy]

83. Pallasea Bate, 1862

446. Pallasea (Homalogammarus) brandtii brandtii (Dybowsky, 1874) B

447. Pallasea (Homalogammarus) brandtii flaviceps Dorogostaisky, 1922 B

448. Pallasea (Homalogammarus) brandtii tenera Sowinsky, 1915 B

449. Pallasea (Babr) baikali baikali Stebbing, $1899 \quad$ B

450. Pallasea (Babr) baikali inermis Sowinsky, $1915 \quad$ B

451. Pallasea (Babr) baikali nigromaculata Dorogostaisky, 1922 B

452. Pallasea (Pallasea) cancelloides (Gerstfeldt, 1858) B

453. Pallasea (Pallasea) cancellus angarensis Dorogostaisky, $1916 \quad$ BE

454. Pallasea (Pallasea) cancellus cancellus (Pallas, 1767) B

-Oniscus cancellus Pallas, 1772 (original combination) [primary monotypy, designated by Barnard, Barnard, 1983]

455. Pallasea (Pallasea) cancellus gerstfeldtii (Dybowsky, 1874) B

456. Pallasea (Pallasea) grubii arenicola Dorogostaisky, 1922 B

457. Pallasea (Pallasea) grubii grubii (Dybowsky, 1874) B

458. Pallasea (Pallasea) kesslerii (Dybowsky, 1874) B B

459. Pallasea (Pallasea) maligna Tachteew, $2000 \quad$ B

460. Pallasea (Pallasea) quadrispinosa Sars, 1867 BE

syn.: Pallasea laevis Ekman, 1923 $3^{12}$ BE

461. Pallasea (Pentagonurus) dybowskii dybowskii Stebbing, 1899 B

462. Pallasea (Pentagonurus) dybowskii fluminalis Tachteew, $2000 \quad$ BE

463. Pallasea (Pentagonurus) viridis (Garjajew, 1901) B

84. Parapallasea Stebbing, 1899

464. Parapallasea borowskii borowskii (Dybowsky, 1874) B

465. Parapallasea borowskii sitnikovae Tachteew, $2000 \quad$ B

466. Parapallasea borowskii wosnessenskii Dorogostaisky, 1922 B

467. Parapallasea lagowskii (Dybowsky, 1874) B

-Gammarus lagowskii Dybowsky, 1874 (original combination) [designated by Dybowski, 1924] 
468. Parapallasea puzyllii nigra (Garjajew, 1901)

syn.: Palicarinus puzyllii carinulata (Dorogostajsky, 1922)

469. Parapallasea puzyllii puzyllii (Dybowsky, 1874)

B

syn.: Palicarinus puzyllii (Dybowsky, 1874)

85. Poekilogammarus Stebbing, 1899

470. Poekilogammarus (Bathygammarus) lydiae (Bazikalova, 1935)

471. Poekilogammarus (Bathygammarus) semenkewitschi okunewae Tachteew, 1995

B

472. Poekilogammarus (Bathygammarus) semenkewitschi semenkewitschi (Sowinsky, 1915)

473. Poekilogammarus (Bathygammarus) unguisetosus (Sowinsky, 1915) B

474. Poekilogammarus (Inobsequentus) galini Tachteew, 2000

475. Poekilogammarus (Onychogammarus) araneolus (Dybowsky, 1874)

476. Poekilogammarus (Onychogammarus) crassimanus Sowinsky, 1915

477. Poekilogammarus (Onychogammarus) dorogostajskii dorogostajskii Tachteew, 1995

478. Poekilogammarus (Onychogammarus) dorogostajskii pygoacanthus Tachteew, 1995

479. Poekilogammarus (Onychogammarus) ephippiatus (Dybowsky, 1874)

480. Poekilogammarus (Onychogammarus) erinaceus Tachteew, 1992

481. Poekilogammarus (Onychogammarus) longipes Bazikalova, 1945

482. Poekilogammarus (Onychogammarus) megonychoides Bazikalova, 1945

483. Poekilogammarus (Onychogammarus) megonychus megonychus Sowinsky, 1915

484. Poekilogammarus (Onychogammarus) megonychus perpolitus Tachteew, 2000

485. Poekilogammarus (Onychogammarus) pictoides Sowinsky, 1915

486. Poekilogammarus (Poekilogammarus) pictus (Dybowsky, 1874)

-Gammarus pictus Dybowsky, 1874 (original combination) [designated by Bousfield, 1977]

487. Poekilogammarus (Poekilogammarus) sukaczewi Sowinsky, 1915

488. Poekilogammarus (Rostrogammarus) rostratus amblyops Bazikalova, 1945

489. Poekilogammarus (Rostrogammarus) rostratus brevirostris Bazikalova, 1945

490. Poekilogammarus (Rostrogammarus) rostratus longirostris Bazikalova, 1945

491. Poekilogammarus (Rostrogammarus) rostratus rostratus Sowinsky, 1915

492. Poekilogammarus (Rostrogammarus) talitrus orchestes (Dybowsky, 1874)

493. Poekilogammarus (Rostrogammarus) talitrus talitrus (Dybowsky, 1874)

494. Poekilogammarus (Variogammarus) curvirostris Bazikalova, 1945

495. Poekilogammarus (Variogammarus) rectirostris Bazikalova, 1945

20. FAMILY PONTOGAMMARIDAE Bousfield, 1977 [10 G 41 S]

86. Compactogammarus Stock, 1974

496. Compactogammarus compactus (G.O. Sars, 1895)

-Niphargoides compactus G.O. Sars, 1895 (original combination) [primary monotypy]

87. Dikerogammarus Stebbing, 1899

497. Dikerogammarus aralychensis (Birstein, 1932)

498. Dikerogammarus bispinosus Martynov, 1925

PCE (?)

499. Dikerogammarus caspius (Pallas, 1771)

PCE

PCE

500. Dikerogammarus fluviatilis Martynov, 1919

PCE

501. Dikerogammarus gruberi Mateus et Mateus, $1990 \quad$ PCE

502. Dikerogammarus haemobaphes (Eichwald, 1841) PCE

-Gammarus haemobaphes Eichwald, 1841 (original combination) [designated by Stock, 1974]

503. Dikerogammarus oskari Birstein, 1945

504. Dikerogammarus palmatus Martynov, 1925

PCE

505. Dikerogammarus villosus (Sowinsky, 1894)

PCE

PCE

88. Niphargogammarus Birstein, 1945

506. Niphargogammarus aequimanus (G.O. Sars, 1895)

507. Niphargogammarus borodini (G.O. Sars, 1897)

PCE

508. Niphargogammarus intermedius (Carausu, 1943)

PCE

509. Niphargogammarus quadrimanus (G.O. Sars, 1895)

PCE

PCE 
- Gammarus quadrimanus G.O. Sars, 1895 (original combination) [designated by Barnard, Barnard, 1983]

89. Niphargoides G.O. Sars, 1894

510. Niphargoides boltovskoi Derzhavin et Pjatakova, 1968

PCE

511. Niphargoides caspius (Grimm in G.O. Sars, 1894)

PCE

-Niphargus caspius Grimm in G.O. Sars, 1894 (original combination) [primary monotypy]

512. Niphargoides corpulentus G.O. Sars, 1894

PCE

90. Obesogammarus Stock, 1974

513. Obesogammarus acuminatus Stock, Mirzajani, Vonk, Naderi, Kiabi, 1998

514. Obesogammarus crassus (Grimm in G.O. Sars, 1894)

PCE

515. Obesogammarus obesus (G.O. Sars, 1894)

-Gammarus obesus G.O. Sars, 1894 (original combination) [designated by Stock, 1974]

516. Obesogammarus olvianus (Sowinsky, 1904)

517. Obesogammarus platycheir (G.O. Sars, 1896)

PCE

PCE

PCE

PCE

91. Pandorites G.O. Sars, 1895

518. $\bullet$ Pandorites podoceroides G.O. Sars, 1895 [primary monotypy]

PCE

92. Paraniphargoides Stock, 1974

519. Paraniphargoides derzhavini (Pjatakova, 1962)

PCE

520. Paraniphargoides grimmi (G.O. Sars, 1896)

PCE

521. Paraniphargoides motasi (Carausu, 1943)

PCE

-Niphargoides motasi Carausu, 1943 (original combination) [designated by Stock, 1974]

93. Pontogammarus Sowinsky, 1904

522. Pontogammarus abbreviatus (G.O. Sars, 1894)

PCE

523. Pontogammarus aestuarius Derzhavin, 1924

PCE

524. Pontogammarus borceae Carausu, 1943

PCE

525. Pontogammarus maeoticus (Sowinsky, 1894)

PCE

526. Pontogammarus robustoides (Grimm in G.O. Sars, 1894)

PCE

- Gammarus robustoides (Grimm in G.O. Sars, 1894 (original combination) [designated by Stock, 1974]

syn.: Gammarus aralensis caspius Sars, 1896

527. Pontogammarus sarsi (Sowinsky, 1898)

PCE

528. *Pontogammarus setosus (Schaferna, 1914) ${ }^{13}$

529. Pontogammarus weidemanni (G.O. Sars, 1896)

94. Stenogammarus Martynov, 1924

530. Stenogammarus (Stenogammarus) carausui Derzhavin et Pjatakova, $1962 \quad$ PCE

531. Stenogammarus (Stenogammarus) compressus (G.O. Sars, 1894) PCE

532. Stenogammarus (Stenogammarus) deminutus (Stebbing, 1906) PCE

533. Stenogammarus (Stenogammarus) macrurus (G.O. Sars, 1894) PCE

-Gammarus macrurus G.O. Sars, 1894 (original combination) [designated by Stock, 1974]

534. Stenogammarus (Stenogammarus) micrurus Derzhavin et Pjatakova, $1996 \quad$ PCE

535. Stenogammarus (Stenogammarus) similis (G.O. Sars, 1894) PCE

536. Stenogammarus (Wolgagammarus) dzjubani Mordukhai-Boltovskoi et Ljakhov, 1972 PCE

95. Turcogammarus Karaman et Barnard, 1979

Type species: Obesogammarus turcarum Stock, 1974 [designated by Karaman, Barnard, 1979]

537. *Turcogammarus aralensis (Uljanin, 1875) ${ }^{14}$

PCE

Gammarus aralensis Uljanin, 1875 (original combination)

96. Uroniphargoides Stock, 1974

538. Uroniphargoides spinicaudatus (Carausu, 1943)

PCE

-Niphargoides spinicaudatus Carausu, 1943 (original combination) [primary monotypy] 


\section{FAMILY PONTOPOREIIDAE Dana, 1853 [2 G 2 S]}

97. Pontoporeia Krøyer, 1842

539. •Pontoporeia femorata Krøyer, 1842 [primary monotypy]

SPC

syn.: Pontoporeia furcigera Bruzelius, 1859

syn.: Pontoporeia sinuata Ekman, 1913

syn.: Pontoporeia ekmani Bulycheva, 1936

98. Monoporeia Bousfield, 1989

540. Monoporeia affinis (Lindström, 1855)

-Pontoporeia affinis Lindström, 1855 (original combination)

AOE

syn.: Monoporeia microphthalma (G.O. Sars, 1896)

FAMILY PSEUDOCRANGONYCTIDAE Holsinger, 1989 [2 G 10 S]

99. Procrangonyx Schellenberg, 1934

Type species: Eocrangonyx japonicus Ueno, 1930, primary monotypy.

541. Procrangonyx primoryensis (Stock et Jo, 1990)

SPC

syn.: Procrangonyx stygoedincus Sidorov et Holsinger, 2007

100. Pseudocrangonyx Akatsuka et Komai, 1922

Type species: Pseudocrangonyx shikokunis Akatsuka et Komai, 1922, designated by Barnard, Barnard [1983]

542. Pseudocrangonyx birsteini Labay, 2001

SPC

543. *Pseudocrangonyx bohaensis (Derzhavin, 1927)

SPC

544. Pseudocrangonyx camtschaticus Birstein, 1955

SPC

545. Pseudocrangonyx elenae Sidorov, 2011

SPC

546. Pseudocrangonyx febras Sidorov, 2009

SPC

547. Pseudocrangonyx holsingeri Sidorov et Gontcharov, 2013

SPC

548. Pseudocrangonyx korkishkoorum Sidorov, 2006

SPC

549. Pseudocrangonyx kseniae Sidorov, 2012

$\mathrm{SPC}$

550. Pseudocrangonyx levanidovi Birstein, 1955

SPC

551. Pseudocrangonyx relictus Labay, 2001

SPC

552. Pseudocrangonyx susunaensis Labay, 2001

$\mathrm{SPC}$

553. Pseudocrangonyx sympatrycus Sidorov et Gontcharov, 2013

SPC

554. Pseudocrangonyx tiunovi Sidorov et Gontcharov, 2013

SPC

FAMILY TALITRIDAE Rafinesque, 1815 [5 G 9 S]

101. Deshayesorchestia Ruffo in Tafani et al., 2004

555. Deshayesorchestia deshayesii (Audouin, 1826)

BSE

- Orchestia deshayesii Audouin, 1826 (original combination) [designated by Tafani et al., 2004]

syn.: Orchestoidea deshayesi (Audouin, 1826)

syn.: Talorchestia deshayesii (Audouin, 1826)

syn.: Orchestia deshayesi Heller, 1866

102. Orchestia Leach, 1814

Type species: Cancer (Gammarus) littoreus Montagu, 1808, primary monotypy.

556. Orchestia bottae Milne-Edwards, 1840

SPC

557. Orchestia cavimana Heller, 1865

BSE

558. Orchestia gammarellus (Pallas, 1766)

WP, BSE

559. Orchestia solifuga Iwasa, 1939

SPC 
103. Paciforchestia Bousfield, 1982

Type species: Parorchestia klawei Bousfield, 1961, designated by Bousfield, 1982.

560. Paciforchestia pyatakovi (Derzhavin 1937)

SPC

104. Platorchestia Bousfield, 1982

561. Platorchestia platensis (Krøyer, 1845)

WP, BSE

- Orchestia platensis Krøyer, 1845 (original combination) [designated by Bousfield, 1982]

562. Platorchestia monodi Mateus, Mateus et Afonso, 1986

WP, BSE

563. Platorchestia joi Stock et Biernbaum, 1994

SPC

syn.: Talorchestia crassicornis Derzhavin, 1937

564. Platorchestia pachypus (Derzhavin, 1937)

SPC

565. *Platorchestia zachsi (Derzhavin, 1937)

SPC

105. Talitrus Latreille, 1802

566. Talitrus saltator (Montagu, 1808)

-Gammarus saltator Montagu, 1808 (original combination) [primary monotypy]

H, BSE

syn.: Oniscus locusta Pallas, 1766

syn.: Talitrus locusta Latreille, 1802

syn.: Talitrus littoralis Leach, 1814

syn.: Talitrus locustra Sars, 1890

106. Traskorchestia Bousfield, 1982

Type species: Orchestia traskiana Stimpson, 1857, designated by Bousfield [1982].

567. * Traskorchestia ditmari (Derzhavin, 1923)

SPC

568. Traskorchestia ochotensis (Brandt, 1851)

107. Trinorchestia Bousfield, 1982

569. Trinorchestia trinitatis (Derzhavin, 1937)

SPC

-Orchestoidea trinitatis Derzhavin, 1937 (original combination) [designated by Bousfield, 1982]

FAMILY URISTIDAE Hurley, 1963 [1 G 5 S ]

108. Onisimus Boeck, 1871

Type species: Anonyx litoralis Krøyer, 1845, designated by Boeck [1876].

570. Onisimus birulai (Gurjanova, 1929) AOE

571. Onisimus botkini Birula, 1897 AOE

572. Onisimus caspius (Grimm in G.O. Sars, 1896) PCE

573. Onisimus litoralis (Krøyer, 1845) AOE

574. Onisimus platyceras (Grimm in G.O. Sars, 1896) PCE

Incertae sedis: [2 G $7 \mathrm{~S}$ ]

Presumably belongs to the genus Eulimnogammarus:

575. Eulimnogammarus (?) abyssalis Sowinsky, 1915

B

576. Eulimnogammarus (?) curvimanus Sowinsky, 1915

Presumably belongs to the genus Heterogammarus:

577. Heterogammarus (?) bifasciatus (Dybowsky, 1874) B

578. Heterogammarus (?) ignotus (Dybowsky, 1874) B B

579. Heterogammarus (?) incertus Sowinsky, 1915

Presumably belongs to the family Micruropodidae:

109. Genus Profundalia Kamaltynov, 2001

580. Profundalia tenuis (Bazikalova, 1945)

-Eulimnogammarus tenuis Bazikalova, 1945 [primary monotypy] 
Presumably belongs to the family Pallaseidae:

110. Genus Polyacanthisca Bazikalova, 1937

581. •Polyacanthisca calceolata Bazikalova, 1937 [primary monotypy]

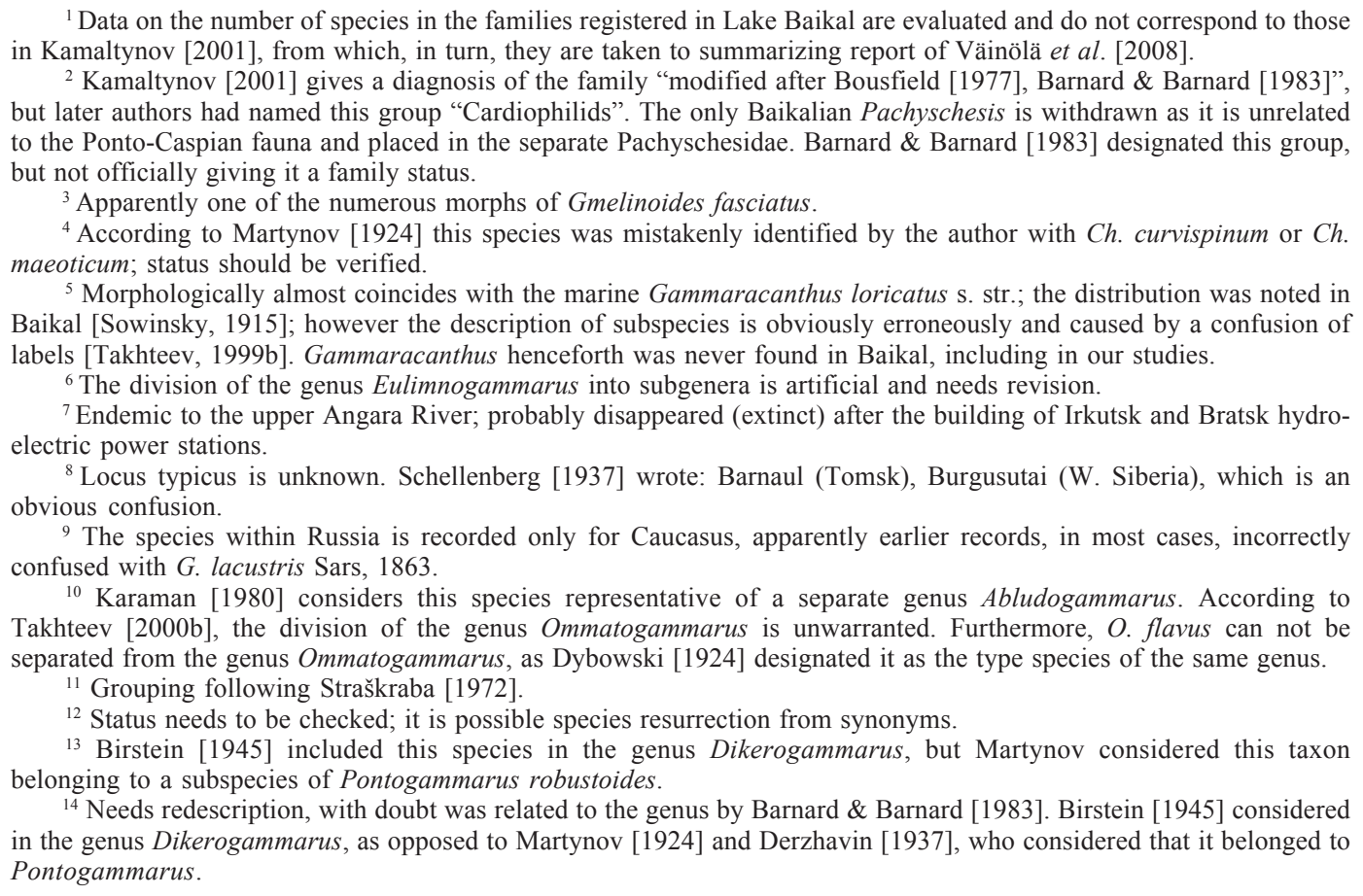

The species composition of the amphipod fauna was studied to a different degree in various Russian regions, often irregularly. Historically, the northwestern region, the Volga Basin, the Russian part of the Altai Mountain Ridge, Lake Baikal, and the southern part of the Russian Far East were relatively better studied.

Seven genera out of the 110 identified (or $6.4 \%$ ) belong to the paleolimnic complex (the genus Pseudocrangonyx presumably attributed to this group). It is the underground amphipods from the families Crangonyctidae and Pseudocrangonyctidae which are the most ancient freshwater inhabitants [Sidorov, Gontcharov, 2015].

The mesolimnic complex includes 44 genera (or $40.0 \%$ ). Its almost all families and genera are from Lake Baikal, with one possible exception (the genus Gmelinoides). It is not inconceivable that the mesolimnic age is typical for all Baikalian families, and to the family Gammaridae and the genus Gammarus in particular. Genetic data suggest the speciation of freshwater representatives of the genus Gammarus in the Eocene [Hou et al., 2011].

The main ecological feature of amphipods from the paleolimnic and, mostly, the mesolimnic complex is adaptation to stable environments. They are present in groundwater, at large depths of Lake Baikal, and in the cold mountain streams. Of course, there are exceptions, such as ubiquistic Gammarus lacustris that is adapted to a broad temperature and salinity range.

The freshwater Lake Baikal is, in fact, an oceanic water-body based on a variety of characteristics: geological, geomorphological, hydrophysical, and by the taxonomic and functional ecological composition and structure of its biota. Baikal can be regarded as a miniature ocean model. The abiotic and biotic factors of endemic Baikal Lake fauna evolution were discussed by many authors [Takhteev, 2000b, c; Takhteev et al., 2003; Baikalogy, 2012].

The Baikal amphipod fauna belonging to the mesolimnic complex is unique (Table 4) across the entire biosphere. Lake Baikal has the water surface area of $31500 \mathrm{~km}^{2}$ (slightly more than $0.000006 \%$ of the area of the Earth's surface, 510200 million $\mathrm{km}^{2}$ ), and its maximum depth is about $1640 \mathrm{~m}$, averaging about 700 $\mathrm{m}$. According to our estimates, 276 species and 78 subspecies of amphipods (totaling 354), distributed among 41 genera and 7 families, are known. Only one species in the lake does not have a Baikalian origin: Gammarus lacustris (is an accidental species in the lake from other water-bodies, and does not survive). There is evidence that another species, Gammarus dabanus, inhabitant of the mountain watercourses of Khamar-Daban Ridge [Takhteev, Mekhanikova, 2000; 
Table 3. Alien amphipods in continental waters of Russia. Таблица 3. Чуждые виды амфипод в континентальных водах России.

\begin{tabular}{|c|c|c|c|}
\hline Species & $\begin{array}{c}\text { Historical } \\
\text { range }\end{array}$ & $\begin{array}{c}\text { Distribution in Russia outside the } \\
\text { historic range }\end{array}$ & First and recent published records \\
\hline Amathillina cristata & $\mathrm{PC}$ & $\begin{array}{l}\text { South European Russia, R. Volga, } \\
\text { Don }\end{array}$ & $\begin{array}{l}\text { Ioffe \& Maximova [1968], Jażdżewski } \\
\text { [1980], Zinchenko et al. [2008] }\end{array}$ \\
\hline $\begin{array}{l}\text { Chelicorophium } \\
\text { curvispinum }\end{array}$ & $\mathrm{PC}$ & $\begin{array}{l}\text { South European Russia, R. Volga, } \\
\text { Neva, Narva, Lake Ladoga, Baltic } \\
\text { Sea in Kaliningrad and Leningrad } \\
\text { reg. }\end{array}$ & $\begin{array}{l}\text { Nikolaev [1963], Jażdżewski [1980], } \\
\text { Ezhova et al. [2005], Zinchenko et al. } \\
\text { [2008], Malyavin et al. [2008], Kurashov } \\
\text { et al. [2010], Berezina } \text { et al. [2011], } \\
\text { Zinchenko \& Kurina [2011] }\end{array}$ \\
\hline $\begin{array}{l}\text { Chelicorophium } \\
\text { maeoticum }\end{array}$ & $\mathrm{PC}$ & $\begin{array}{l}\text { South European Russia, R. Volga, } \\
\text { Don }\end{array}$ & $\begin{array}{l}\text { Filinova et al. [2008], Zinchenko et al. } \\
\text { [2008], Zinchenko \& Kurina [2011] }\end{array}$ \\
\hline $\begin{array}{l}\text { Chelicorophium } \\
\text { sowinskyi }\end{array}$ & $\mathrm{PC}$ & South European Russia, R. Don & $\begin{array}{l}\text { Ioffe [1968], Mordukhai-Boltovskoi et al. } \\
\text { [1969] }\end{array}$ \\
\hline $\begin{array}{l}\text { Chelicorophium } \\
\text { mucronatum }\end{array}$ & $\mathrm{PC}$ & South European Russia, R. Don & Mordukhai-Boltovskoi et al. [1969] \\
\hline Chelicorophium robustum & $\mathrm{PC}$ & South European Russia, R. Don & Mordukhai-Boltovskoi et al. [1969] \\
\hline $\begin{array}{l}\text { Pontogammarus } \\
\text { robustoides }\end{array}$ & $\mathrm{PC}$ & $\begin{array}{l}\text { South European Russia, R. Don, } \\
\text { Volga, Baltic Sea in Kaliningrad and } \\
\text { Leningrad reg., Lake Ladoga }\end{array}$ & $\begin{array}{l}\text { Ioffe \& Maximova [1968], Berezina \& } \\
\text { Panov [2003], Zinchenko et al. [2008], } \\
\text { Kurashov \& Barbashova [2010], } \\
\text { Zinchenko \& Kurina [2011], own data }\end{array}$ \\
\hline $\begin{array}{l}\text { Pontogammarus } \\
\text { abbreviatus }\end{array}$ & $\mathrm{PC}$ & South European Russia, R. Volga & Zinchenko et al. [2008] \\
\hline Pontogammarus sarsi & $\mathrm{PC}$ & South European Russia, R. Volga & $\begin{array}{l}\text { Borodich [1976], Zinchenko et al. } \\
\text { [2008], Filinova et al. [2008] }\end{array}$ \\
\hline Turcogammarus aralensis & $\mathrm{PC}+$ Aral & South European Russia, R. Don & Pjatakova \& Tarasov [1996] \\
\hline Dikerogammarus caspius & $\mathrm{PC}$ & $\begin{array}{l}\text { South European Russia, R. Don, } \\
\text { Volga }\end{array}$ & $\begin{array}{l}\text { Filinova et al. [2008]; } \\
\text { Zinchenko et al. [2008], Zinchenko \& } \\
\text { Kurina [2011], own data }\end{array}$ \\
\hline $\begin{array}{l}\text { Dikerogammarus } \\
\text { haemobaphes }\end{array}$ & $\mathrm{PC}$ & $\begin{array}{l}\text { South European Russia, R. Don, } \\
\text { Volga }\end{array}$ & $\begin{array}{l}\text { Mordukhai-Boltovskoi [1960], Borodich } \\
\text { \& Lyakhov [1983], L'vova et al. [1996], } \\
\text { Bakanov [2003], Berezina [2007a], } \\
\text { Zinchenko \& Kurina [2011] }\end{array}$ \\
\hline Dikerogammarus villosus & $\mathrm{PC}$ & South European Russia, R. Volga & $\begin{array}{l}\text { Mordukhai-Boltovskoi [1960], } \\
\text { Zinchenko et al. [2008] }\end{array}$ \\
\hline $\begin{array}{l}\text { Dikerogammarus } \\
\text { fluviatilis }\end{array}$ & PC & $\begin{array}{l}\text { South European Russia, R. Don, } \\
\text { Volga }\end{array}$ & $\begin{array}{l}\text { Filinova et al. [2008], Zinchenko et al. } \\
\text { [2008] }\end{array}$ \\
\hline Iphigenella acanthopoda & $\mathrm{PC}$ & South European Russia & Zinchenko et al. [2008] \\
\hline Obesogammarus obesus & $\mathrm{PC}$ & $\begin{array}{l}\text { R Volga (incl. upper reservoirs), } \\
\text { South European Russia }\end{array}$ & $\begin{array}{l}\text { Mordukhai-Boltovskoi \& Dzyuban } \\
\text { [1976], Zinchenko et al. [2008], } \\
\text { Zinchenko \& Kurina [2011] }\end{array}$ \\
\hline Obesogammarus crassus & $\mathrm{PC}$ & $\begin{array}{l}\text { South European Russia, R. Don, } \\
\text { Volga, Baltic Sea in Kaliningrad } \\
\text { reg. }\end{array}$ & $\begin{array}{l}\text { Mordukhai-Boltovskoi [1979], Ezhova et } \\
\text { al. [2005], Zinchenko et al. [2008], own } \\
\text { data }\end{array}$ \\
\hline $\begin{array}{l}\text { Echinogammarus } \\
\text { warpachowskyi }\end{array}$ & $\mathrm{PC}$ & $\begin{array}{l}\text { South European Russia, R, Volga; } \\
\text { Baltic Sea in Kaliningrad and } \\
\text { Leningrad reg. }\end{array}$ & $\begin{array}{l}\text { Voronin \& Yermokhin [2004], Orlova et } \\
\text { al. [2006], Filinova et al. [2008], } \\
\text { Berezina et al. [2011], Zinchenko \& } \\
\text { Kurina [2011], own data }\end{array}$ \\
\hline Echinogammarus ischnus & $\mathrm{PC}$ & $\begin{array}{l}\text { South European Russia, R. Volga; } \\
\text { Baltic Sea in Kaliningrad reg. }\end{array}$ & $\begin{array}{l}\text { Mordukhai-Boltovskoi [1960], Ezhova et } \\
\text { al. [2005], Berezina et al. [2011], } \\
\text { Zinchenko \& Kurina [2011], own data }\end{array}$ \\
\hline $\begin{array}{l}\text { Stenogammarus }(W .) \\
\text { dzjubani }\end{array}$ & $\mathrm{PC}$ & South European Russia, R. Volga & $\begin{array}{l}\text { Voronin \& Yermokhin [2004], } \\
\text { Zinchenko et al. [2008], Zinchenko \& } \\
\text { Kurina [2011] }\end{array}$ \\
\hline $\begin{array}{l}\text { Stenogammarus }(S .) \\
\text { macrurus }\end{array}$ & $\mathrm{PC}$ & South European Russia, R. Volga & Zinchenko et al. [2008] \\
\hline $\begin{array}{l}\text { Stenogammarus }(S .) \\
\text { similis }\end{array}$ & $\mathrm{PC}$ & South European Russia, R. Volga & Zinchenko et al. [2008] \\
\hline $\begin{array}{l}\text { Shablogammarus } \\
\text { chablensis }\end{array}$ & $\mathrm{PC}$ & South European Russia & $\begin{array}{l}\text { Voronin \& Yermokhin [2004], } \\
\text { Zinchenko \& Kurina [2011] }\end{array}$ \\
\hline Orchestia cavimana & PC + Med. & $\begin{array}{l}\text { Basin of R .Narva,Baltic Sea in } \\
\text { Leningrad and Kaliningrad reg. }\end{array}$ & $\begin{array}{l}\text { Kotta [2000], Berezina et al. [2011], own } \\
\text { data }\end{array}$ \\
\hline
\end{tabular}


Table 3 (contituing) Таблица 3 (продолжение)

\begin{tabular}{|l|c|l|l|}
\hline Platorchestia platensis & CT & $\begin{array}{l}\text { Basins of R .Narva, Baltic Sea in } \\
\text { Leningrad reg, NW Russia }\end{array}$ & Spicer \& Janas [2006], own data \\
\hline Gmelinoides fasciatus & B & $\begin{array}{l}\text { Basins of R. Narva, Neva, Volga, Ural } \\
\text { including small and large lakes; } \\
\text { Reservoirs in Siberia }\end{array}$ & $\begin{array}{l}\text { Borodich [1979], Zadoenko et al. [1985], } \\
\text { Tarasov [1995], Berezina [2007c], own } \\
\text { data }\end{array}$ \\
\hline Micruropus wohlii & B & Reservoirs, R. Volga, Siberia & $\begin{array}{l}\text { Voronin \& Yermokhin [2004], Filinova } \\
\text { et al. [2008] }\end{array}$ \\
\hline Micruropus possolskii & B & $\begin{array}{l}\text { Basins of R. Narva, Neva, Volga, Ural, } \\
\text { Ob; Lake Ladoga; large and small } \\
\text { lakes, reservoirs }\end{array}$ & $\begin{array}{l}\text { Zadoenko } \text { et al. [1985], Tarasov [1995], } \\
\text { Viser [1981], Barbashova et al. [2013], } \\
\text { own data }\end{array}$ \\
\hline Gammarus tigrinus & NA & $\begin{array}{l}\text { Basins of R. Narva, Neva, Baltic Sea } \\
\text { in Leningrad and Kaliningrad reg. }\end{array}$ & $\begin{array}{l}\text { Nikolaev [1963], Berezina [2007b], } \\
\text { Berezina et al. [2011], own data }\end{array}$ \\
\hline Gammarus lacustris & $\begin{array}{c}\text { Loc., E, } \\
\text { S }\end{array}$ & $\begin{array}{l}\text { lakes of European Russia, Siberia and } \\
\text { Kamtchatka }\end{array}$ & $\begin{array}{l}\text { Deksbakh [1952], Bekman [1954], } \\
\text { Kurenkov [1967], Timm \& Timm [1993], } \\
\text { Kozlov [2013] }\end{array}$ \\
\hline Monoporeia affinis & Loc., E & lakes of European Russia, Siberia & Greze [1958] \\
\hline Pallasea quadrispinosa & Loc., E & lakes of north European Russia & Gordeev [1954] \\
\hline
\end{tabular}

Notes: PC — Ponto-Caspian, B — Baikalian, NA — North-American, CT — Circumtropical, Med. — Mediterranean, Aral — Aral Sea, S - Siberia, E - European, Loc. — Local.

Table 4. Correlation of amphipod life-forms with zoogeographic groups for native continental amphipods. Таблица 4. Корреляция жизненных форм амфипод с зоогеографическими группами для нативных континенентальных амфипод.

\begin{tabular}{|c|c|c|c|c|c|c|c|c|c|c|c|c|}
\hline \multirow{2}{*}{\multicolumn{2}{|c|}{ Class }} & \multirow{2}{*}{ Group } & \multirow{2}{*}{ Taxa } & \multicolumn{9}{|c|}{ Geographic attribution $^{1}$} \\
\hline & & & & $\mathbf{H}$ & WP & $\mathbf{C}$ & SPC & CA & B & BE & BSE & AOE \\
\hline 1 & Terrestrial & amphibionts & Talitridae & & & & + & & & & & \\
\hline 2 & Pelagic & mysidiformes & Macrohectopus & & & & & & + & & & \\
\hline \multirow[t]{11}{*}{3} & \multirow{11}{*}{ Benthic } & \multirow{2}{*}{ true benthics } & \multirow{2}{*}{$\begin{array}{l}\text { Eulimnogammarus (part.) } \\
\dagger \text { Fluviogammarus }\end{array}$} & & & & & & + & & & \\
\hline & & & & & & & & & & + & & \\
\hline & & \multirow{3}{*}{ nectobenthic } & \multirow{3}{*}{$\begin{array}{l}\text { Acanthogammarus } \\
\text { Gammaracanthidae } \\
\text { Parapallaseinae }\end{array}$} & & & & & & + & & & \\
\hline & & & & & & & & & & & & + \\
\hline & & & & & & & & & + & & & \\
\hline & & \multirow{2}{*}{ phytophilic } & \multirow{2}{*}{$\begin{array}{l}\text { Pallasea (part.) } \\
\text { Micruropus vortex }\end{array}$} & & & & & & + & $+?$ & & \\
\hline & & & & & & & & & + & & & \\
\hline & & \multirow{4}{*}{ variable modes } & \multirow{4}{*}{$\begin{array}{l}\text { Anisogammaridae } \\
\text { Gammarus } \\
\text { Gmelinoides } \\
\text { Baikalogammarus }\end{array}$} & & & & + & & & & & \\
\hline & & & & + & + & + & + & + & & & + & \\
\hline & & & & & & & & & + & + & & \\
\hline & & & & & & & & & & & & \\
\hline \multirow[t]{2}{*}{4} & \multirow{2}{*}{ Benthopelagic } & \multirow{2}{*}{ scavengers } & \multirow{2}{*}{$\begin{array}{l}\text { Ommatogammarus } \\
\text { Polyacanthisca }\end{array}$} & & & & & & + & & & \\
\hline & & & & & & & & & + & & & \\
\hline \multirow[t]{3}{*}{5} & \multirow{3}{*}{ Symbiont } & \multirow[b]{2}{*}{ sponge symbiotic } & \multirow{2}{*}{$\begin{array}{l}\text { Brandtia parasitica } \\
\text { Eulimnogammarus } \\
\text { violaceus }\end{array}$} & & & & & & + & & & \\
\hline & & & & & & & & & + & & & \\
\hline & & amphipod parasites & Pachyschesidae & & & & & & + & & & \\
\hline \multirow[t]{6}{*}{6} & \multirow{6}{*}{ Subterranean } & \multirow{4}{*}{ stout body } & \multirow{4}{*}{$\begin{array}{l}\text { Niphargidae } \\
\text { Amurocrangonyx } \\
\text { Crangonyx } \\
\text { Ganigamoera }\end{array}$} & & & + & & & & & & \\
\hline & & & & & & & + & & & & & \\
\hline & & & & & + & & & & & & & \\
\hline & & & & & & & + & & & & & \\
\hline & & \multirow{2}{*}{ slender body } & \multirow{2}{*}{$\begin{array}{l}\text { Pseudocrangonyctidae } \\
\text { Stygobromus }\end{array}$} & & & & + & & & & & \\
\hline & & & & & & & & + & & & & \\
\hline \multirow[t]{2}{*}{7} & Semi- & & Synurella & & + & + & + & & & & & \\
\hline & subterranean & stout body & Lyurella & & & + & & & & & & \\
\hline
\end{tabular}

${ }^{1}$ Designation of groups see in section "Material and methods". Data about the lifestyle of amphipods in Baikal Lake according Takhteev [1996, 2000b, c], Baikalogy [2012]. 
Mekhanikova, 2009], can reach the mouths of rivers and the water edge of Lake Baikal [Kamaltynov, 2009]. Even excluding the subspecies in Lake Baikal, about $4.3 \%$ of the world's amphipod fauna and $45.3 \%$ of the inland amphipods are present there [Takhteev, 2000b]. However, taking into consideration the stygobionts (as can be calculated from the data given by Väinölä et al. [2008]), the share of the Baikalian fauna species accounts for $28.5 \%$ of all known freshwater amphipods.

The neolimnic complex includes 59 genera (or $53.6 \%$ ). It consists of the superfamilies Hadzioidea, Eusiroidea, Oedicerotoidea, Lysianassoidea, Phoxocephaloidea, Talitroidea and Corophiidea. Members of this complex are found in relative proximity to the sea coasts, in the riverine estuaries as well as in different relict lakes that had a connection with the Ocean or formed directly during marine transgression (in particular, Monoporeia and Gammaracanthus).

The diversity of life forms of the amphipods is more abundant in ancient Lake Baikal (see Table 4). In particular, only in this lake that the life forms are native to freshwater basins as mysidiformic pelagobionts (Macrohectopus branickii), benthopelagic scavengers (six species and subspecies of the genus Ommatogammarus and Polyacanthisca calceolata), symbionts of sponges (Brandtia spp., and Eulimnogammarus violaceus) and large amphipod parasites (fam. Pachyschesidae, no less than 16 species). However, their ecological analogs exist in the oceans, which once again confirms the oceanic ecosystem type characteristic of Lake Baikal.

As in the other regions of the planet, the class of terrestrial life forms icludes amphipods of the family Talitridae that are the amphibiotic inhabitants of the sea coastal regions.

The subterranean aquatic amphipod fauna of Russia is not rich in species (totaling 35 species). However, their zoogeographical heterogeneity is of interest; the underground representatives belong to four biogeographic groups (see Table 4). Four groups could be identified, which differ markedly in their origin and evolution: 1) Holarctic group of the paleolimnic complex Crangonyx-Synurella; 2) a group of the Western Palearctic Niphargidae, related to the mesolimnic complex; 3) a group of the Far Eastern Pseudocrangonyctydae of unknown origin [Sidorov, Gontcharov, 2015], and 4) a group of the neolimnic Far Eastern Ganigamoera [Sidorov, 2010].

The amphipod biodiversity of Russian continental waters is quite impressive when compared with the rest of the planet. However unique it may be is still rather understudied and underappreciated. Only in Lake Baikal, according to forecasts, at least one-third of the species is undescribed. The underground fauna is poorly understood or even unknown in some regions to date. A good proof of this is the recent discovery in Siberia of two new species of the genus Stygobromus, whose main centre of diversity is located in North America [Sidorov et al., 2010]. In conclusion, some of the species (possibly remains) as yet unknown may inhabit the mountainous regions of the Central Asian Foldbelt.

First Russian record:

Eogammarus itotomikoae Tomikawa et al., 2006 [this publication] SPC

List of the extinct species:

It should be noted that the group of species, emigrants from Lake Baikal (Baikalian escapee), has greatly suffered owing to the hydropower construction on the Angara river, and due to switchover of the reservoirs from the river regime to the lake regime. The four rheophilous Fluviogammarus species mentioned below have disappeared from the hydrobiological samples in recent years.

Fluviogammarus angarensis Bazikalova, 1945

Fluviogammarus brachyurus (Dorogostaisky, 1916)

Fluviogammarus intermedius Bazikalova, 1945

Fluviogammarus larviformis Dorogostaisky, 1916

ACKNOWLEDGMENTS. The authors are grateful to Prof. Nina G. Bogutskaya (earlier in Zoological Institute RAS, St. Petersburg, Russia) for useful advice on the nomenclature problems with the Lake Baikal amphipods, Dr. Cene Fišer (University of Ljubljana, Slovenia) for valuable advice on Niphargidae taxonomy, and Dr. Ksenia Semenchenko (Institute of Biology and Soil Science, Vladivostok, Russia) for the final preparation of the manuscript. We would also like to thank Prof. Gordan S. Karaman (Montenegrin Academy of Sciences and Arts, Podgorica, Montenegro) for critically reading and commenting on an early draft of the manuscript. Our special thanks are due to Vasiliy G. Mikhalkovsky (V.B. Sochava Institute of Geography SB RAS, Irkutsk, Russia) for revising and improving English style.

The study was supported by the grants from the Russian Foundation for Basic Research (\#\# 09-0498544, 13-04-00614), from the Presidium of the Russian Academy of Sciences, Far Eastern Branch (\#\# 13III-B-06-047, 14-I-P30-01), and from the Zoological Institute of the Russian Academy of Sciences (theme \#01201351192).

\section{References}

Baikalogy. 2012. / O.T. Rusinek, V.V. Takhteev, D.P. Gladkochub, T.V. Khodzher, N.M. Budnev (eds.). Novosibirsk: Nauka. 1114 p. [in Russian].

Bakanov A.I. 2003. [Current state of benthos in the upper Volga River in Yaroslavl region] // Biologiya vnutrennikh vod. No.1. P.81-88 [in Russian].

Barbashova M.A., Malyavin S.A., Kurashov E.A. 2013. First finding of baikalian amphipod Micruropus possolskii Sowinsky, 1915 (Amphipoda, Crustacea) in Lake Ladoga // Russian Journal of Biological Invasions. No.3. P.16-23 [in Russian, with English summary].

Barnard J.L., Barnard C.M. 1983. Freshwater Amphipoda of the world. Mt. Vernon, Virginia. 830 p.

Barnard J.L., Karaman G.S. 1991. The Families and Genera of Marine Gammaridean Amphipoda (Except Marine Gammaroids). Part $1 / /$ Records of the Australian Museum. Supplement. Vol.13. P.1-417. 
Bate C.S. 1862. Catalogue of the specimens of amphipodous Crustacea in the collection of the British Museum. London: Taylor \& Francis. 399 p.

Bazikalova A.Ya. 1935. [To the systematics of Baikal Lake Amphipoda] // Trudy Baikalskoi Limnologicheskoi Stantsii. Vol.6. P.31-52 [in Russian].

Bazikalova A.Ya. 1945. [Les Amphipodes du Baïkal] // Trudy Baikalskoi Limnologicheskoi Stantsii. Vol.11. P.1-440 [in Russian, with French summary].

Bazikalova A.Ya. 1948. [Notes about amphipods of Baikal Lake] // Trudy Baikalskoi Limnologicheskoi Stantsii. Vol.12. P.2032 [in Russian].

Bazikalova A.Ya. 1971. [Bottom fauna] // Limnologiya pridel'tovikh prostranstv Baikala. Selenginsky rayon. Trudy Limnolog icheskogo Instituta Sibirskogo Otdeleniya AN SSSR. Vol.12(32). P.95-114 [in Russian].

Bekman M.Y. 1954. [Biology of Gammarus lacustris Sars from waterbodies near Lake Baikal] // Trudy Baikalskoi Limnologicheskoi Stantsii. Vol.14. P.263-311 [in Russian].

Berezina N.A. 2007a. Invasions of alien amphipods (Crustacea: Amphipoda) in aquatic ecosystems of North-Western Russia: pathways and consequences // Hydrobiologia. Vol.590. P.1529. http://dx.doi.org/10.1007/s10750-007-0753-z

Berezina N.A. 2007b. Expansion of the North American amphipod Gammarus tigrinus Sexton, 1939 to the Neva Estuary (easternmost Baltic Sea) // Oceanologia. Vol.49. No.1. P.129-135.

Berezina N.A. 2007c. Changes in aquatic ecosystems of the northwestern Russia after introduction of Baikalian amphipod Gmelinoides fasciatus // F. Gherardi (ed.). Biological invaders in inland waters: profiles, distribution, and threats. Dordrecht: Springer. P.479-493. http://dx.doi.org/10.1007/978-1-40206029-8_26

Berezina N.A., Panov V.E. 2003. Establishment of new gammarid species in the eastern Gulf of Finland (Baltic Sea) and their effects on littoral communities // Proceedings of Estonian Academy of Sciences, Biology, Ecology. Vol.52. P.284-304

Berezina N.A., Petryashev V.V., Razinkovas A., Lesutiene J. 2011 Alien malacostracan crustaceans in the Eastern Baltic Sea: pathways and consequences // B.S. Galil, P.F. Clark, J.T. Carlton (eds.). In the wrong place - alien marine crustaceans: distribution, biology and impacts. Dordrecht: Springer. P.301-322. http://dx.doi.org/10.1007/978-94-007-0591-3 10

Birstein J.A. 1935. [About origin of marine crustaceans in rivers of Ponto-Caspian basin] // Zoologicheskii Zhurnal. Vol.14. No.4. P.749-761 [in Russian].

Birstein J.A. 1945. [Revision of the system of Caspian Gammaridae] // Doklady AN SSSR. Vol.50. P.517-520 [in Russian].

Boeck A. 1876. De skandinaviske og arktiske amphipoder. Christiania: A.W. Brøgger. 137 p.

Borodich N.D. 1976. [Representatives of Ponto-Caspian fauna in reservoirs of the middle and lower Volga River in 1971-1974] // Biologiya vnutrennikh vod. Informatsionnyi byulleten'. No.29. P.35-36 [in Russian].

Borodich N.D. 1979. [Baikalian amphipod Gmelinoides fasciatus (Stebb.) (Amphipoda, Gammaridae) in Kuibyshev Reservoir // Zoologicheskii Zhurnal. Vol.58. No.6. P.920-921 [in Russian].

Bousfield E.L. 1977. A new look at the systematics of gammaroidean amphipods of the world // Crustaceana. Suppl.4. P.282316 .

Bousfield E.L. 1982. Amphipoda. Gammaridea // S.P. Parker (ed.) Synopsis and classification of living organisms. New York: McGraw-Hill. Vol.2. P.254-285.

Bousfield E.L., Chevrier A. 1996. The amphipod family Oedicerotidae on the Pacific coast of North America. I. The Monoculodes \& Synchelidium generic complexes: systematics and distributional ecology // Amphipacifica. Vol.2. No.2. P.75-148.

Bousfield E.L., Hoover P.W. 1997. The amphipod superfamily Corophioidea on the Pacific coast of North America. Part V. Family Corophiidae: Corophiinae, new subfamily. Systematics and distributional ecology // Amphipacifica. Vol.2. No.3. P.67-139.

Chernov Yu.I. 1975. [Natural zoning and land fauna]. Moscow:
Mysl‘. 222 p. [in Russian].

Chevreux E., Fage L. 1925. Amphipodes // Faune de France. T.9. $488 \mathrm{p}$.

Daneliya M.E., Kamaltynov R.M., Kontula T., Väinölä R. 2009. Systematics of the Baikalian Babr (Crustacea: Amphipoda: Pallaseidae) // Zootaxa. Vol.2276. P.49-68.

Daneliya M.E., Väinölä R. 2014. Five subspecies of the Dorogostaiskia parasitica complex (Dybowsky) (Crustacea: Amphipoda: Acanthogammaridae), epibionts of sponges in Lake Baikal // Hydrobiologia. Vol.739. No.1. P.95-117. http:// dx.doi.org/10.1007/s10750-013-1671-x

Dedyu I.I. 1963. [On role of amphipods in geographical dispersion of dreissenid mollusks] // Izvestiya Academii nauk Moldavskoi SSR. No.5. P.4-65 [in Russian].

Deksbakh N.K. 1952. [The amphipod Gammarus lacustris in waterbodies of the Ural region] // Trudy Vsesoyuznogo Gidrobiologicheskogo Obshchestva. Vol.4. P.187-199 [in Russian].

Derzhavin A.N. 1937. [Talitridae of the Soviet coast if the Japan Sea] // Issledovaniya morei SSSR. Vol.23. P.87-99 [in Russian, with English summary].

Derzhavin A.N. 1948. [New gammarids species from the Caspian Sea] // E.N. Pavlovskyi, V.I. Shadin (eds.). Pamiaty akademika Sergeia Aleksandrovicha Zernova. Moscow-Leningrad: USSR Academy of Sciences Publishing House. P.280-286 [in Russian].

Dybowsky B. 1874. Beiträge zur näheren Kenntnis der in dem Baikal-See vorkommenden niederen Krebse aus der Gruppe der Gammariden // Herausgegeben von der Russischen Entomologischen Gesellschaft zu St. Petersburg. St. Petersburg: Buchdruckerei von W. Besobrasoff und Comp. 190 S., 14 Taf.

Dybowsky B. 1875. [Gammaroidea of the Baikal Lake] // Izvestiya Sibirskogo otdeleniya Russkogo Geograficheskogo obshchestva. Vol.6. Nos.1/2. P.10-80 [in Russian].

Dybowski B. 1924. Baicalogammaridea. - Gammariden des Baikalsees. 1 Teil. Limnophili Baicalogammarini. (Die den Boden des Sees bewohnenden Formen) // Bulletin International de l'Academie Polonaise des Sciences et des Letters. Classe des Sciences Mathematiques et Naturelles. Serie B: Sciences naturelles, supplementaire. Cracovie. S.1-92 [in German].

Dybowski B. 1926/1927. Synoptisches Verzeichnis mit kurzer Besprechung der Gattungen und Arten dieser Abteilung der Bakalflohkrebse // Bulletin International de l'Academie Polonaise des Sciences et des Letters. Classe des Sciences Mathematiques et Naturelles. Serie B: Sciences naturelles, supplementaire. Cracovie. S.1-77 [in German].

Ezhova E., Żmudziński L., Maciejewska K. 2005. Long-term trends in the macrozoobenthos of the Vistula Lagoon, southeastern Baltic Sea. Species composition and biomass distribution // Bulletin of the Sea Fisheries Institute, Gdynia. No.1(164). P.55-73.

Filinova E.I., Malinina Y.A., Shlyakhtin G.V. 2008. Bioinvasions in Macrozoobenthos of the Volgograd Reservoir // Russian Journal of Ecology. Vol.39. No.3. P.193-197. http://dx.doi.org/ $10.1134 / \mathrm{S} 1067413608030077$

Gordeev O.N. 1954. [Introduction of crustaceans as food for fish to lakes of the Karelian-Finish SSR] // Materialy soveshchaniya po probleme povysheniya rybnoi produktivnosti vnutrennikh vodoenov Karelo-Finskoi ASSR, 11-12 Marta 1953, Petrozavodsk. P.52-161 [in Russian].

Grosswald M.G. 1998. Late-Weichselian ice sheets in Arctic and Pacific Siberia // Quaternary International. Vol.45-46. P.3-18.

Greze V.N. 1958. [Relict mysis (Mysis oculata v. relicta) and pontoporean amphipod (Pontoporeia affinis) as objects of acclimatization] // Zoologicheskii Zhurnal. Vol.37. No.10. P.1449-1461 [in Russian].

Gurjanova E.F. 1953. [New additions to the far Eastern fauna of the marine amphipods] // Trudy Zoologicheskogo Instituta AN SSSR. Leningrad. Vol.13. P.216-241 [in Russian].

Hou Zh., Sket B., Fišer C., Li S.-Q. 2011. Eocene habitat shift from saline to freshwater promoted Tethyan amphipod diversiûcation // Proceedings of the National Academy of Sciences of the United States of America. Vol.108. No.35. P.14533-14538. http://dx.doi.org/10.1073/pnas.1104636108

Hou Zh., Li J., Li S.-Q. 2013. Ten new Gammarus species (Crusta- 
cea: Amphipoda: Gammaridae) from Yunnan-Guizhou Plateau, China // Zootaxa. Vol.3687. No.1. P.1-95. http:// dx.doi.org/10.11646/zootaxa.3687.1.1

Ioffe T.I. 1968. [Rationale and results of acclimatization of invertebrates in large reservoirs on the Volga and Don Rivers] // Akklimatizatsiya ryb i bespozvonochnykh v vodoemakh SSSR. Moscow: Nauka. P.148-155 [in Russian].

Ioffe T.I., Maximova L.P. 1968. [Biology of some crustaceans used for introduction in reservoirs] // Izvestiya GosNIORKh. Vol.67. P.87-104 [in Russian].

Jażdżewski K. 1980. Range extension of some gammaridean species in European inland waters caused by human activity // Crustaceana. No.6. P.84-107.

Jeschke J.M., Strayer D.L. 2005. Invasion success of vertebrates in Europe and North America // Proceedings of the National Academy of Sciences of the United States of America. Vol.102. No.20. P.7198-7202.

Kamaltynov R.M. 1992. [On the presented state of systematics of the Lake Baikal amphipods (Crustacea, Amphipoda)] // Zoologicheskii Zhurnal. Vol.71. No.6. P.24-31 [in Russian, with English summary].

Kamaltynov R.M. 1999. On the higher classification of Lake Baikal amphipods // Crustaceana. Vol.72. No.8. P.933-944. http:// dx.doi.org/10.1163/156854099503834

Kamaltynov R.M. 2001. Amphipoda: Gammaroidea // O.A. Timoshkin (ed.). Annotirovannyi spisok fauny ozera Baikal i ego vodosbornogo basseina [Index of animal species inhabiting Lake Baikal and its catchment area]. Vol.1: Ozero Baikal [Lake Baikal], Book 1. Novosibirsk: Nauka. P.572-831 [in Russian and English].

Kamaltynov R.M. 2009. Amphipoda: Gammaroidea in Angara and Yenisei River // O.A. Timoshkin (ed.). Annotirovannyi spisok fauny ozera Baikal i ego vodosbornogo basseina [Index of animal species inhabiting Lake Baikal and its catchment area]. Vol.2: Vodoemy i vodotoki Vostochnoi Sibiri I Severnoi Mongolii [Basins and Channels in the south of East Siberia and North Mongolia], Book 1. Novosibirsk: Nauka. P.297-329 [in Russian and English].

Karaman G.S. 1977. Revision of the genus Carinurus Sow., 1915 from Baikal Lake (Fam. Gammaridae). Contribution to the Knowledge of the Amphipoda 81 // Poljoprivreda i Shumarstvo. Vol.23. No.1. P.33-52.

Karaman G.S. 1980. New genus of family Gammaridae from Baikal Lake, Abludogammarus, n. gen. with reference to genus $\mathrm{Om}$ matogammarus Stebb. Contribution to the Knowledge of the Amphipoda 108. // Montenegrin Academy of Sciences and Arts, Glasnik of the Section of Natural Sciences. No.3. P.149167.

Karaman G.S., Barnard J.L. 1979. Classificatory revision in Gammaridean Amphipoda (Crustacea). Part 1. // Proceedings of the Biological Society of Washington. Vol.92. No.1. P.106-165.

Karaman G.S., Pinkster S. 1977. Freshwater Gammarus species from Europe, North Africa and adjacent regions of Asia (Crustacea-Amphipoda), 1. Gammarus pulex-group and related species // Bijdragen tot de Dierkunde. Vol.47. No.1. P.1-97.

Ketmaier V., De Matthaeis E. 2010. Allozymes and mtDNA reveal two divergent lineages in Orchestia cavimana (Amphipoda: Talitridae) // Journal of Crustacean Biology. Vol.30. No.2. P.307-311. http://dx.doi.org/10.1651/09-3162.1

Khlebovich V.V. 1974. [Critical salinity of biological processes] Leningrad: Nauka Publishing House. 256 p. [in Russian].

Kotta J. 2000. First record of the talitrid amphipod Orchestia cavimana in the northern Baltic Sea // Proceedings of the Estonian Academy of Sciences. Biology, Ecology. Vol.49. No.2. P.221-224.

Kozlov O. 2013. Gammarus lacustris relocation in Ishim plain lakes: an impostor in the habitable home // Book of abstracts of the $15^{\text {th }}$ International Colloquium on Amphipoda, 2-7 September 2013, Szczawnica, Poland. P.54.

Kurashov E.A., Barbashova M.A. 2008. First record of the invasive Ponto-Caspian amphipod Pontogammarus robustoides G.O. Sars, 1894 from Lake Ladoga, Russia // Aquatic Invasions. Vol.3. No.2. P.253-256. http://dx.doi.org/10.3391/ai.2008.
3.2 .18

Kurashov E.A., Barbashova M.A., Panov V.E. 2010. First finding of Ponto-Caspian invasive amphipod Chelicorophium curvispinum (G.O. Sars, 1895) (Amphipoda, Crustacea) in Lake Ladoga // Russian Journal of Biological Invasions. Vol.1. No.4. P.282287. http://dx.doi.org/10.1134/S2075111710040053

Kurenkov I.I. 1967. [List of aquatic invertebrates of the inland waters of Kamchatka] // Izvestiya TINRO. Vol.57. P.202-224 [in Russian].

Latreille P.A. 1810. Considérations générales sur l'ordre natural des animaux composant les classes des Crustacés, des Arachnides et des Insectes; avec un tableau méthodique de leurs genres, disposés en familles. Paris: F. Schoell. 444 p.

L'vova A.A., Palii A.V., Sokolova N.Y. 1996. [Ponto-Caspian invaders in the River Moskva and in the city Moscow] // Zoologicheskii Zhurnal. Vol.75. No.8. P.1273-1274 [in Russian].

Lyubarsky G.Yu. 1996. [Archaetype, style and ranking in biological systematics] // Sbornik trudov Zoologicheskogo Muzeya MGU. Moscow: KMK Scientific Press Ltd. Vol.35. 436 p. [in Russian]

Malyavin S.A., Berezina N.A., Hwang J.-S. 2008. [About record of Chelicorophium curvispinum Sars, 1895 (Amphipoda: Crustacea) in the Gulf of Finland of the Baltic Sea] // Zoologicheskii Zhurnal. Vol.87. No.6. P.643-649 [in Russian].

Martinson G.G. 1967. [The problem of origin of Baikal Lake fauna] // Zoologicheskii Zhurnal. Vol.46. No.10. P.1594-1598 [in Russian, with English summary].

Martynov A.V. 1924. [On some interesting Malacostraca from fresh-waters of Europaean Russia] // Russian Hydrobiological Journal. Vol.3. No.8/10. P.210-216 [in Russian, with English summary].

Martynov A.V. 1930. [The amphipod fauna of Teletskoye Lake and its origin] // Izvestiya Gosudarstvennogo Gidrologicheskogo Instituta. Vol.29. P.95-128 [in Russian].

Mekhanikova I.V. 2009. [Morphology of Gammarus dabanus Tachteew et Mekhanikova, 2000 (Crustacea, Amphipoda, Gammaridea) from mountain streams of Khamar-Daban Ridge (Transbaikalia)] // Invertebrate Zoology. Vol.6. No.1. P.21-31 [in Russian, with English summary].

Miers E.J. 1875. Descriptions of new species of Crustacea collected at Kerguelen's Island by the Rev. A.E. Eaton // Annals and Magazine of Natural History. Ser.4. Vol.16. No.91. P.73-76. http://dx.doi.org/10.1080/00222937508681124

Mordukhai-Boltovskoi F.D. 1960. [Caspian fauna in the Azov and Black Seas basin]. Moskva-Leningrad: USSR Academy of Sciences Publishing House. 287 p. [in Russian]

Mordukhaj-Boltovskoi F.D. 1979. Biogeography of the Volga // F.D. Mordukhaj-Boltovskoj (ed.). The River Volga and its life. Monograph Biology. Vol.33. P.346-366. http://dx.doi.org/ 10.1002/iroh.19800650315

Mordukhai-Boltovskoi F.D., Dzyuban N.A. 1976. [Chandes in composition and distribution of fauna of the Volga river as a result of anthropogenic impacts] // Biologicheskaja productivnost'v basseyne reki Volga. Leningrad: Nauka. P.67-82 [in Russian].

Mordukhai-Boltovskoi F.D., Greze I.I., Vasilenko S.V. 1969. [Order Amphipoda Latreille, 1816-1817] // V.A. Vodjanickij (ed.). Jpredelitel' fauny Chernogo i Azovskogo morei [Guide to the fauna of the Black Sea and Azov Sea]. Vol.2. Svobodnozhivushchie bespozvonochnye. Rakoobraznye. Kiev: Naukova Dumka. P.440-524 [in Russian].

Nikolaev I.I. 1963. [New invaders in the fauna and flora of the North Sea and Baltic] // Zoologicheskii Zhurnal. Vol.42. No.1. P.20-27 [in Russian].

Orlova M.I., Telesh I.V., Berezina N.A., Antsulevich A.E., Maximov A.A., Litvinchuk L.F. 2006. Effects of nonindigenous species on diversity and community functioning in the eastern Gulf of Finland (Baltic Sea) // Helgoland Marine Research. No.2. P.98-105. http://dx.doi.org/10.1007/s10152-006-0026-7

Pinkster S. 1973. The Echinogammarus berilloni-group, a number of predominantly Iberian amphipod species (Crustacea) // Bijdragen tot de Dierkunde. Vol.43. No.1. P.1-38.

Pjatakova G.M., Tarasov A.G. 1996. Caspian Sea amphipods: biodi- 
versity, systematic position and ecological peculiarities of some species // International Journal of Salt Lake Research. Vol.5. No.1. P.63-79. http://dx.doi.org/10.1007/BF01996036

Rachalewski M., Banha F., Grabowski M., Anastácio P.-M. 2013. Ectozoochory as a possible vector enhancing the spread of an alien amphipod Crangonyx pseudogracilis // Hydrobiologia. Vol.717. No.1. P.109-117.

Sars G.O. 1894. Crustacea caspia. Contributions to the knowledge of the carcinological fauna of the Caspian Sea. Part 3. Amphipoda // Bulletin de l'Academie Imperiale des Sciences de St.-Petersbourg. Vol.1. No.2. P.179-223, 8 plates.

Sars G.O. 1896. Crustacea caspia. Contributions to the knowledge of the carcinological fauna of the Caspian Sea. Amphipoda // Bulletin de 1'Academie Imperiale des Sciences de St.-Petersbourg. Vol.4. No.5. P.421-489, 12 plates.

Schellenberg A. 1937. Kritische Bemerkungen zur Systematik der Süsswassergammariden // Zoologischer Jahrbücher. Abteilung für Systematik. Bd.69. S.469-516.

Shoemaker C.R. 1941. A new genus and a new species of Amphipoda from the Paciûc coast of North America // Proceedings of the Biological Society of Washington. Vol.54. P.183-186.

Sidorov D.A. 2010. A new subgenus of eusirid amphipod (Crustacea: Amphipoda: Eusiridae) from subterranean waters and springs of the Eastern Sikhote-Alin Mountain Ridge, with comments on the morphology of sternal humps, genital papillae and pleopods // Zootaxa. Vol.2518. P.1-31.

Sidorov D.A., Chertoprud E.S., Semikolennykh A.A., Shavrina E.V. 2011. Mysterious Pallasea (Crustacea: Amphipoda) from the caves Golubinsky Gap and Kitezh // Karstovye sistemy Severa v menyayushcheisya srede. Sbornik tezisov Mezhdunarodnoi konferentsii, posvyashchennoi 300-letiyu so dnya rozhdeniya M.V. Lomonosova, 5-10 Sent. 2011. Golubino-Pinega, Arkhangelsk Region, Russia. P.85-87 [in Russian].

Sidorov D.A., Gontcharov A.A. 2015. Preliminary analysis of phylogenetic relationships of the Asian-Pacific endemial subterranean amphipod genus Pseudocrangonyx among families and genera of crangonyctoidean amphipods inferred by partial LSU rDNA gene sequences // Zoological Sciences. Vol.32. Issue 2. P.178-182.

Sidorov D.A., Holsinger J.G., Takhteev V.V. 2010. Two new species of the subterranean amphipod genus Stygobromus (Amphipoda: Crangonyctidae) from Siberia, with new data on Stygobromus pusillus (Martynov) and remarks on morphology and biogeographic relationships // Zootaxa. Vol.2478. P.41-58.

Sowinsky V.K. 1915. [Amphipoda of Lake Baikal] // Zoologicheskie issledovaniya Baikala. Kiev. Vol.9. No.1. 381 p., 37 plates [in Russian and German].

Spicer J.I., Janas U. 2006. The beachflea Platorchestia platensis (Krøyer, 1845): a new addition to the Polish fauna (with a key to Baltic talitrid amphipods) // Oceanologia. Vol.48. No.2. P.287-295.

Starobogatov Ya.I. 1970. [Fauna of molluscs and zoogeographical zoning of the continental waterbodies of the globe]. Leningrad: Nauka. 372 p. [in Russian].

Stebbing T.R.R. 1899. Amphipoda from the Copenhagen Museum and other sources. Part 2 // Transactions of the Linnean Society of London. Second series. Zoology. Vol.7. No.2. P.25-45, plates 6-14. http://dx.doi.org/10.1111/j.1096-3642.1897. tb00400.x

Stock J. 1967. A revision of the European species of the Gammarus locusta-group (Crustacea, Amphipoda) // Zoologische Verhandelingen. Bd.90. P.3-56.

Stock J.H. 1968. A revision of the European species of the Echinogammarus pungens-group (Crustacea, Amphipoda) // Beaufortia. Vol.16. No.211. P.13-78.

Stock J.H. 1969. Members of Baikal amphipod genera in European waters, with description of a new species, Eulimnogammarus macrocarpus, from Spain // Proceedings of the Koninklijke Nederlandse Akademie van Wetenschappen. Series C. Biological and medical sciences. Vol.72. No.1. P.66-75.

Stock J.H. 1974. The systematics of certain PontoCaspian Gammaridae (Crustacea, Amphipoda) // Mitteilungen aus dem Zoologischen Staatsinstitut und Zoologischen Museum, Hamburg. Bd.70. P.75-95.
Stock J. 1995. Gammarus and Chaetogammarus (Crustacea, Amphipoda) from Macaronesia // Boletim do Museu Municipal do Funchal. No.45(247). P.41-52.

Straškraba M. 1972. Les groupements des espèces du genre Niphargus (sensu lato) // S. Ruffo (ed.), Actes du I ${ }^{\text {er }}$ Colloque Internationale sur le genre Niphargus, Verona, 15-16 Aprile 1969. Museo Civico di Storia Naturale di Verona. P.85-90.

Tafani B., Ugolini A., Bazzicalupo M., Mengoni A., Ruffo S. 2004. Phylogenetic relationships among Mediterranean sandhoppers // Journal of Natural History. Vol.38. No.4. P.499-508.

Takhteev V.V. 1992a. [Carinogammarus cinnamomeus (Dybowsky, 1874) = Echinogammarus borealis Sowinsky, 1915, syn. n.] // Vestnik zoologii. Kiev. No.4. P.72 [in Russian].

Takhteev V.V. (=Tachteew W.W.) 1992b. [Poekilogammarus erinaceus sp. n. - new species of Baikal sand-hoppers (Amphipoda, Gammaridae)] // Zoologicheskii Zhurnal. Vol.71. No.2. P.150-153 [in Russian, with English summary].

Takhteev V.V. 1993. [A note on a "lost" species of the Baikal gammarids (Amphipoda, Gammaridae)] // Zoologicheskii Zhurnal. Vol.72. No.1. P.152-153 [in Russian, with English summary].

Takhteev V.V. (=Tachteew V.V.) 1995. The gammarid genus Poekilogammarus Stebbing, 1899, in Lake Baikal, Siberia (Crustacea Amphipoda Gammaridea) // Arthropoda Selecta. Vol.4. No.1. P.7-64.

Takhteev V.V. 1996. On the ecology of a rare species of amphipod, Polyacanthisca calceolata (Crustacea, Amphipoda) of Lake Baikal, with reference to parallelism in the evolution of the baikal and oceanic deepwater faunas // Hydrobiological Journal. Vol.32. No.2. P.107-109.

Takhteev V.V. 1997. The gammarid genus Plesiogammarus Stebbing, 1899, in Lake Baikal, Siberia (Crustacea Amphipoda Gammaridea) // Arthropoda Selecta. Vol.6. Nos.1/2. P.31-54.

Takhteev V.V. 1998. [Parasite-hosts relationship between endemic Baikal amphipods] // Vzaimootnosheniya parazita i khozyaina. Vserossiyskaya mauchnaya konferentsiya, 8-10 Dek. 1998, Moskva. Tezisy dokladov. P.64 [in Russian].

Takhteev V.V. 1999a. [Revision of the genus Odontogammarus (Crustacea, Amphipoda, Gammaridae) from Lake Baikal] // Zoologicheskii Zhurnal. Vol.78. No.7. P.796-810 [in Russian, with English summary].

Takhteev V.V. 1999b. [The problem of a hypothetical elements of the common genesis in Baikal and Arctic Ocean amphipod faunas] // Byulleten' Moskovskogo Obshchestva Ispytatelei Prirody. Otdel Biologicheskiy. Vol.104. No.4. P.3-11 [in Russian, with English summary].

Takhteev V.V. 2000a. [Addition to revision of the genus Poekilogammarus (Crustacea, Amphipoda, Gammaridea) from Lake Baikal] // Zoologicheskii Zhurnal. Vol.79. No.6. P.649-661 [in Russian, with English summary].

Takhteev V.V. 2000b. [Esseyes on the amphipods of Lake Baikal: systematics, comparative ecology, evolution]. Irkutsk: Irkutsk State University Press. 350 p. [in Russian].

Takhteev V.V. 2000c. Trends in the evolution of Baikal amphipods and evolutionary parallels with some marine malacostracan faunas // A. Rossiter, H. Kawanabe (eds.). Advances in ecological research. Vol.31. Ancient lakes: biodiversity, ecology and evolution. London: Academic Press. P.197-220. http:// dx.doi.org/10.1016/S0065-2504(00)31013-3

Takhteev V.V. 2009. [Amphipods (Amphipoda) in thermal and mineral springs from the northern part of Baikal Lake region] // A.S. Pleshanov (ed.). Biota vodoemov Baikal'skoy riftovoy zony. Irkutsk: Irkutsk State University Press. P.123-130 [in Russian].

Takhteev V.V. 2010. [Archaetypical method of approach to the systematization of rich taxonomic groups (an example of endemic amphipods from Baikal Lake)] // Problemy ekologii. Chteniya pamyati Prof. M.M. Kozhova (Irkutsk, 20-25 Sentyabrya 2010 g.). Irkutsk: Irkutsk State University Press. P.262 [in Russian].

Takhteev V.V. 2012. [Problems in macrosystematics of amphipods of Baikal Lake] // Aktual'nye problemy isucheniya rakoobraznykh kontinental'nykh vod. Sbornik lektsiy i dokladov Mezhdunarodnoi shkoly-konferentsii. Institut Biologii Vnu- 
trennik Vod im. I.D. Papanina RAN, Borok, 5-9 Noyabrya 2012 g. Kostroma: Kostroma Printing House. P.293-296 [in Russian].

Takhteev V.V., Levashkevich A.M. 2006. [To the systematic of the genus Garjajewia (Crustacea, Amphipoda) from Lake Baikal with description of a new subspecies] // Zoologicheskii Zhurnal. Vol.85. No.12. P.1422-1432 [in Russian, with English summary].

Takhteev V.V., Mekhanikova I.V. 2000. [New species of amphipods (Crustacea Amphipoda) from the mountain streams of Khamar-Daban Ridge] // V.V. Takhteev (ed.). Issledovaniya vodnykh ekosistem Vostochnoi Sibiri. Irkutsk: Irkutsk State University Press. P.115-123 [in Russian, with English summary].

Takhteev V.V., Mekhanikova I.V., Govorukhina E.B. 2003. [Ecological mechanisms of an endemic evolution of sand hoppers (Crustacea Amphipoda) of the Lake Baikal] // Siberian Ecological Journal. No.3. P.305-310 [in Russian, with English summary].

Takhteev V.V., Sidorov D.A. 2012. [Diversity and distribution of amphipod in continental waters of Northern Eurasia] // Aktual'nye problemy isucheniya rakoobraznykh kontinental'nykh vod. Sbornik lektsiy i dokladov Mezhdunarodnoi shkoly-konferentsii. Institut Biologii Vnutrennik Vod im. I.D. Papanina RAN, Borok, 5-9 Noyabrya 2012 g. Kostroma: Kostroma printing house. P.102-107 [in Russian].

Tarasov A.G. 1995. [Crustaceofauna (Malacostraca) of the Ural River] // Zoologicheskii Zhurnal. Vol.74. No.3. P.24-34 [in Russian].

Timm V., Timm T. 1993. The recent appearance of a Baikalian crustacean, Gmelinoides fasciatus (Stebbing, 1899) (Amphipoda, Gammaridae) in Lake Peipsi // Proceedings of the Estonian Academy of Sciences. Biology. Vol.42. No.2. P.144-153.

Tzvetkova N. 1972. [Taxonomy of the genus Gammarus Fabr., with the description of some new species of gammarids (Amphipoda, Gammaridae) from the north-western part of the $\mathrm{Pa}$ - cific Ocean] // Trudy Zoologicheskogo Instituta AN SSSR. Leningrad. Vol.52. P.201-222 [in Russian].

Tzvetkova N.L. 1975. [Coastal gammarids northern and Far East seas USSR and adjacent waters: genera Gammarus, Marinogammarus, Anisogammarus, Mesogammarus (Amphipoda, Gammaridae)]. Leningrad: Nauka. 257 p. [in Russian].

Väinölä R., Witt J.D.S., Grabowski M., Bradbury J.H., Jazdzewski K., Sket B. 2008. Global diversity of amphipods (Amphipoda; Crustacea) in freshwater // Hydrobiologia. Vol.595. P.241255. http://dx.doi.org/10.1007/978-1-4020-8259-7_27

Vizer A.M. 1981. [Results of introductions of Baikalian gammarids in Novosibirsk reservoir] // Rybnoe Khozyaistvo. No.4. P.47-48 [in Russian].

Voronin M.Yu., Yermokhin M.V. 2004. [Stability of the onthogenesis of amphipods (Crustacea, Amphipoda) and the outlook of its usage for biomonitoring of the ecosystems of cooling reservoirs of nuclear power stations] // Povolzhskiy Ekologicheskii Zhurnal. No.2. P.123-131 [in Russian].

Wrześniowski A. 1877. Über die Anatomie der Amphipoden. Protocolle der Sitzungen des Section für Zoologie und vergleichende Anatomie des Versammlung russischer Naturforscher und Ärzte in Warschau in September 1876, mitetheilt von prof. Hoyer // Zeitschrift für wissenschaftliche Zoologie, Leipzig. Bd.28. S.403-404.

Zadoenko I.N., Leis O.A., Grigor'ev V.F. 1985. [Results and perspectives of acclimatization of the Baikalian amphipods in water bodies of the USSR] // Sbornik nauchnykh trudov GosNIORKh. Vol.232. P.30-34 [in Russian].

Zinchenko T.D., Golovatjuk L.V., Zagorskaya E.P., Antonov P.I. 2008. [Distribution of invasive species as a part of the bottom communities in the Kuibyshev water reservoir: analysis of long-term researches] // Izvestiya Samarskogo Nauchnogo Tsentra RAN. Vol.2. 547-558 [in Russian].

Zinchenko T.D., Kurina E.M. 2011. Distribution of alien species in open shallow waters of Saratov reservoir // Russian Journal of Biological Invasions. No.2. P.74-85 [in Russian].

Responsible editor K.G. Mikhailov 